TRANSACTIONS OF THE

AMERICAN MATHEMATICAL SOCIETY

Volume 348, Number 12, December 1996, Pages 4895-4918

S 0002-9947(96)01782-5

\title{
CHARACTERIZATIONS OF KADEC-KLEE PROPERTIES IN SYMMETRIC SPACES OF MEASURABLE FUNCTIONS
}

\author{
V. I. CHILIN, P. G. DODDS, A. A. SEDAEV, AND F. A. SUKOCHEV
}

\begin{abstract}
We present several characterizations of Kadec-Klee properties in symmetric function spaces on the half-line, based on the $K$-functional of J. Peetre. In addition to the usual Kadec-Klee property, we study those symmetric spaces for which sequential convergence in measure (respectively, local convergence in measure) on the unit sphere coincides with norm convergence.
\end{abstract}

\section{INTRODUCTION}

If $\left(E,\|\cdot\|_{E}\right)$ is a normed linear space, then $E$ is said to have the Kadec-Klee property (sometimes called the Radon-Riesz property, or property $(\mathrm{H})$ ) if and only if sequential weak convergence on the unit sphere coincides with norm convergence. As is well known, this property was first studied by J. Radon [Ra] and subsequently by F. Riesz [Ri1], [Ri2] who showed that the classical $L_{p}$-spaces, $1<p<\infty$, have the Kadec-Klee property. Although the space $L_{1}[0,1]$ (with Lebesgue measure) fails to have the Kadec-Klee property, Riesz showed that each of the $L_{p}$-spaces, $1 \leq p<\infty$, has the property that each sequence on the unit sphere that converges almost everywhere converges also in norm.

In this paper, we study properties of Kadec-Klee type in the setting of symmetric (rearrangement-invariant) spaces $E$ on the positive half-line in which sequential weak convergence is replaced by convergence in some natural linear space topology on $E$. In addition to the usual weak topology, we consider the weak topology induced on $E$ by the ideal $\Lambda_{\infty}=L_{1} \cap L_{\infty}$ (which coincides with the weak topology if $E$ has separable dual, and with the weak*-topology if $E$ is the dual of some separable symmetric space) as well as the linear topologies of convergence in measure and local convergence in measure. Each of these linear topologies plays a natural and important role in the study of symmetric spaces. For example, it has been shown by Sedaev [Se1], [Se2] that each separable symmetric space on the positive half-line has an equivalent symmetric norm for which sequential $\sigma\left(E, \Lambda_{\infty}\right)$ convergence on the unit sphere coincides with norm convergence. This is to be compared with the well-known renorming theorem of Kadec [Ka1] that each separable Banach space admits an equivalent locally uniformly convex norm, and therefore has the Kadec-Klee property for some equivalent (Banach space) norm. The analogue and appropriate strengthening of the Kadec renorming theorem in the setting of general Banach lattices with order continuous norm has been given in [DGL], where it is also

Received by the editors October 29, 1994 and, in revised form, May 25, 1995.

1991 Mathematics Subject Classification. Primary 46E30; Secondary 46B20, 46B42.

Key words and phrases. Symmetric spaces, Kadec-Klee properties, Lorentz spaces.

Research of the second and fourth authors was supported by the Australian Research Council. 
stated that each separable symmetric space on a finite interval has an equivalent symmetric locally uniformly convex norm.

While there are a number of conditions which imply the Kadec-Klee property in various settings, there are relatively few criteria for various Kadec-Klee type properties in terms of natural properties of the underlying space, even in spaces of measurable functions. It is the main purpose of the present paper to develop such criteria in the case of symmetric spaces of measurable functions. One of the principal results of this paper (Theorem 2.7) exhibits the relationship in separable symmetric spaces between the Kadec-Klee properties for convergence and local convergence in measure and the Kadec-Klee property with respect to $\Lambda_{\infty}$ and shows that these properties can be characterized in terms of the pre-order induced by the (so-called) $K$-functional of J. Peetre. Symmetric spaces $E$ in which the norm is strictly monotone for this pre-order are said to have strictly $K$-monotone norm. In separable Lorentz spaces, strict $K$-monotonicity of the norm is equivalent to the Kadec-Klee property (Theorem 2.11). On the other hand, for symmetric spaces in which the norm is strictly monotone for the natural (pointwise) ordering, the Kadec-Klee properties for convergence in measure and local convergence in measure can be characterized in terms of local uniform monotonicity properties of the norm (Theorem 3.2). One consequence is that a Lorentz space $\Lambda_{\phi}$ on $[0, \infty)$ has the Kadec-Klee property for local convergence in measure if and only if $\Lambda_{\phi}$ is separable (Theorem 3.3) in contrast to the fact that any Lorentz space $\Lambda_{\phi}$ has the KadecKlee property for convergence in measure (Corollary 1.3). As an application of the main results, Theorem 4.1 shows that in separable rotund dual symmetric spaces, the Kadec-Klee property for the weak*-topology coincides with the Kadec-Klee properties for convergence in measure and local convergence in measure and that each of these properties is equivalent to mid-point local uniform convexity; further, it is not possible in this setting to replace mid-point local uniform convexity by local uniform convexity. We remark finally that the main results of this paper carry over without difficulty to the setting of symmetric spaces on $\sigma$-finite non-atomic measure spaces, but for the sake of simplicity of presentation of the main ideas, we confine our attention to the positive half-line. The authors wish to thank the referee for a very thorough reading of the original manuscript and for a number of helpful suggestions. The authors also wish to thank E. M. Semenov and A. V. Bukhvalov for their comments on earlier versions of this paper.

\section{Preliminaries}

Let $0<\alpha \leq \infty$, and let $m$ denote Lebesgue measure on the real line. A Banach space $\left(E,\|\cdot\|_{E}\right)$ of real-valued measurable functions on the interval $[0, \alpha)$ will be called a symmetric space if (i) $E$ is an ideal lattice (that is, if $y \in E$, and if $x$ is any measurable function on $[0, \alpha)$ with $0 \leq|x| \leq|y|$, then $x \in E$ and $\|x\|_{E} \leq\|y\|_{E}$ ), (ii) $E$ is rearrangement-invariant in the sense that if $y \in E$, and if $x$ is any measurable function on $[0, \alpha)$ with $x^{*}=y^{*}$, then $x \in E$ and $\|x\|_{E}=\|y\|_{E}$. Here, $x^{*}$ denotes the non-increasing, right-continuous rearrangement of $x$ given by

$$
x^{*}(t)=\inf \{s \geq 0: m(\{|x|>s\}) \leq t\}, \quad t>0 .
$$

For basic properties of symmetric spaces and rearrangements, we refer to $[\mathrm{Lu}]$ and to the monographs [KPS], [BS] . We put $L_{1}=L_{1}([0, \alpha), m), L_{\infty}=L_{\infty}([0, \alpha), m)$, and consider the spaces $\Lambda_{\infty}=\Lambda_{\infty}([0, \alpha), m)=L_{1} \cap L_{\infty}$ and $\Lambda_{1}=\Lambda_{1}([0, \alpha), m)=$ 
$L_{1}+L_{\infty}$ with the usual norms. The continuous embeddings

$$
\Lambda_{\infty} \subset E \subset \Lambda_{1}
$$

hold for each symmetric space $E$. We will denote by $E^{\prime}$ the symmetric space associated with $E$. The space $E^{\prime}$ consists of all measurable functions $y$ for which

$$
\|y\|_{E^{\prime}}:=\sup \left\{\int_{0}^{\infty} x(t) y(t) d t: x \in E,\|x\|_{E} \leq 1\right\}<\infty .
$$

If $E^{*}$ denotes the Banach dual of $E$, it is known that $E^{\prime} \subset E^{*}$ and $E^{\prime}=E^{*}$ if and only if the norm $\|\cdot\|_{E}$ is order-continuous, i.e. from $\left\{x_{n}\right\} \subseteq E, x_{n} \downarrow_{n} 0$, it follows that $\left\|x_{n}\right\|_{E} \rightarrow 0$. We note that the norm $\|\cdot\|_{E}$ on the symmetric space $E$ is ordercontinuous if and only if $E$ is separable, in which case $x^{*}(\infty)=\lim _{t \rightarrow \infty} x^{*}(t)=0$ for all $x \in \mathrm{E}$.

We consider on $\Lambda_{1}$ the pre-order generated by the $K$-functional of J. Peetre:

$$
x \prec \prec y \Longleftrightarrow \int_{0}^{t} x^{*}(s) d s \leq \int_{0}^{t} y^{*}(s) d s \text { for all } t>0 .
$$

It is well known (see, for example, $[\mathrm{KPS}]$ ) that $\left(E,\|\cdot\|_{E}\right)$ is an interpolation space for the pair $\left(L_{1}, L_{\infty}\right)$, with interpolation constant 1 , if and only if $x \prec \prec y, y \in E$, implies $x \in E$ and $\|x\|_{E} \leq\|y\|_{E}$. In particular, this is the case if the norm of the symmetric space $E$ is order-continuous.

We recall that the symmetric space $\left(E,\|\cdot\|_{E}\right)$ is said to have the Kadec-Klee property if and only if

$$
x \in E,\left\{x_{n}\right\} \subseteq E, \quad x_{n} \rightarrow x \sigma\left(E, E^{*}\right), \quad\left\|x_{n}\right\|_{E} \rightarrow\|x\|_{E} \Rightarrow\left\|x_{n}-x\right\|_{E} \rightarrow 0 .
$$

If $\sigma\left(E, E^{*}\right)$-convergence is replaced by $\sigma\left(E, \Lambda_{\infty}\right)$-convergence (respectively, convergence in measure, convergence in measure on every set of finite measure), then the symmetric space $\left(E,\|\cdot\|_{E}\right)$ is said to have the Kadec-Klee property with respect to $\Lambda_{\infty}$ (respectively, convergence in measure, local convergence in measure) .

Proposition 1.1. If the symmetric space $\left(E,\|\cdot\|_{E}\right)$ has either the Kadec-Klee property or the Kadec-Klee property for local convergence in measure, then $E$ is separable.

Proof. If $E$ is not separable, then $E$ does not have order-continuous norm. It follows from $[\mathrm{KA}]$, Chapter $10, \S 4$, that there exists a pairwise disjoint sequence $\left\{z_{n}\right\} \subseteq E$ such that $0 \leq z_{n}$ and $\left\|z_{n}\right\|_{E}=1, n=1,2, \ldots$ and $x=\sum_{n=1}^{\infty} z_{n} \in E$. For $f \in E^{*}$ we have $\sum_{n=1}^{\infty}|f|\left(z_{n}\right) \leq|f|(x)$, hence $\left|f\left(z_{n}\right)\right| \leq|f|\left(z_{n}\right) \rightarrow 0$. Therefore, the sequence $\left\{z_{n}\right\}$ converges to 0 weakly; it is clear also that $\left\{z_{n}\right\}$ converges to 0 locally in measure. If we set $x_{n}=x-2 z_{n}, n=1,2, \ldots$, then the sequence $\left\{x_{n}\right\}$ converges to $x$ both weakly and locally in measure. Since $x_{n}^{*}=x^{*}$, it follows that $\left\|x_{n}\right\|_{E}=\|x\|_{E}$ for all $n=1,2, \ldots$, and therefore, by either assumption, it follows that $\left\|x_{n}-x\right\|_{E} \rightarrow 0$. However,

$$
\left\|x_{n}-x\right\|_{E}=2\left\|z_{n}\right\|_{E}=2, \quad n=1,2, \ldots,
$$

and this is a contradiction.

The preceding proposition does not hold if local convergence in measure is replaced by convergence in measure. This is a consequence of the following proposition which implies that any Lorentz space, separable or not, has the Kadec-Klee property for convergence in measure. We first introduce some necessary notation. 
Denote by $\Phi$ the set of concave increasing functions $\phi$ on $[0, \alpha)$ for which $\phi(0)=$ $0=\phi(0+)$. We note that each function $\phi \in \Phi$ is absolutely continuous and $\phi(t)=$ $\int_{0}^{t} \phi^{\prime}(s) d s$ for all $t>0$. Consequently, it is clear that for $\phi, \psi \in \Phi$ the inequality $\phi(t) \leq \psi(t), t>0$, holds if and only if $\phi^{\prime} \prec \prec \psi^{\prime}$. For every $\phi \in \Phi$, we consider the Lorentz space $\left(\Lambda_{\phi},\|\cdot\|_{\Lambda_{\phi}}\right)$ consisting of all $x \in \Lambda_{1}$ for which

$$
\|x\|_{\Lambda_{\phi}}:=\int_{0}^{\alpha} x^{*}(s) \phi^{\prime}(s) d s<\infty .
$$

The symmetric space associated with $\Lambda_{\phi}$ is the Marcinkiewicz space $M_{\phi}$ consisting of all $x \in \Lambda_{1}$ for which

$$
\|x\|_{M_{\phi}}:=\sup _{0<t<\infty}[\phi(t)]^{-1} \int_{0}^{t} x^{*}(s) d s<\infty .
$$

We remark that if $\alpha<\infty$, then $\Lambda_{\phi}$ has order-continuous norm and so is separable. On the other hand, if $\alpha=\infty$, then $\Lambda_{\phi}$ is separable if and only if $\phi(\infty)=$ $\lim _{t \rightarrow \infty} \phi(t)=\infty$. We will need the following result, which is of interest in its own right.

Proposition 1.2. If $x \in \Lambda_{\phi},\left\{y_{n}\right\} \subseteq \Lambda_{\phi}$, and if $\left\{y_{n}\right\}$ converges to 0 in measure, then

$$
\left\|x+y_{n}\right\|_{\Lambda_{\phi}}=\|x\|_{\Lambda_{\phi}}+\left\|y_{n}\right\|_{\Lambda_{\phi}}+o(1) .
$$

Proof. Using [KPS], Theorem II.3.1, we have that

$$
\begin{aligned}
\left\|x+y_{n}\right\|_{\Lambda_{\phi}} & =\int_{0}^{\infty}\left(x+y_{n}\right)^{*} d \phi \geq \int_{0}^{\infty}\left|x^{*}-y_{n}^{*}\right|^{*} d \phi \geq \int_{0}^{\infty}\left|x^{*}-y_{n}^{*}\right| d \phi \\
& \geq \int_{0}^{\delta}\left(y_{n}^{*}-x^{*}\right) d \phi+\int_{\delta}^{\delta^{-1}}\left(x^{*}-y_{n}^{*}\right) d \phi+\int_{\delta^{-1}}^{\infty}\left(y_{n}^{*}-x^{*}\right) d \phi \\
& =\int_{0}^{\infty} y_{n}{ }^{*} d \phi+\int_{0}^{\infty} x^{*} d \phi-2\left(\int_{0}^{\infty} x^{*} \chi_{\delta} d \phi+\int_{\delta}^{\delta^{-1}} y_{n}^{*} d \phi\right) \\
& =\|x\|_{\Lambda_{\phi}}+\left\|y_{n}\right\|_{\Lambda_{\phi}}-\gamma(n, \delta),
\end{aligned}
$$

where $\delta \in(0,1), \chi_{\delta}$ is the indicator function of the set $[0, \delta] \cup\left[\delta^{-1}, \infty\right)$, and

$$
\gamma(n, \delta)=2 \int_{0}^{\infty} x^{*} \chi_{\delta} d \phi+2 \int_{\delta}^{\delta^{-1}} y_{n}^{*} d \phi, \quad n=1,2, \ldots
$$

Thus we have

$$
0 \leq\|x\|_{\Lambda_{\phi}}+\left\|y_{n}\right\|_{\Lambda_{\phi}}-\left\|x+y_{n}\right\|_{\Lambda_{\phi}} \leq \gamma(n, \delta)
$$

If $\phi(\infty)=\infty$, then fixing $\epsilon>0$ and using the order continuity of the norm $\|\cdot\|_{\Lambda_{\phi}}$, choose $\delta \in(0,1)$ such that $2 \int_{0}^{\infty} x^{*} \chi_{\delta} d \phi<2^{-1} \epsilon$. Since $y_{n}$ converges to zero in measure, we have $y_{n}^{*}(t) \rightarrow 0$ for any $t>0$. Hence $\left\{y_{n}^{*}\right\}$ converges to zero uniformly on $\left[\delta, \delta^{-1}\right]$, and so, there exists a natural number $n_{0}$ such that $2 \int_{\delta}^{\delta^{-1}} y_{n}^{*} d \phi<2^{-1} \epsilon$ for $n \geq n_{0}$. Therefore, $\gamma(n, \delta)<\epsilon$ for $n \geq n_{0}$, so the assertion of the lemma is true for separable spaces $\Lambda_{\phi}$.

Assume now that $\phi(\infty)<\infty$. Passing to a subsequence if necessary, we may assume that $\delta_{n}=m\left(\chi_{A_{n}}\right) \rightarrow 0$, where $A_{n}=\left\{\left|y_{n}\right|>n^{-1}\right\}$. If we set $z_{n}=$ 
$y_{n} \chi_{A_{n}}, a_{n}=y_{n}-z_{n}, n=1,2, \ldots$, then

$$
\left\|a_{n}\right\|_{\Lambda_{\phi}} \leq n^{-1} \int_{0}^{\infty} d \phi \leq n^{-1} \phi(\infty), \quad n=1,2, \ldots
$$

and so

$$
\left\|x+y_{n}\right\|_{\Lambda_{\phi}}=\left\|x+z_{n}\right\|_{\Lambda_{\phi}}+o(1)
$$

Observing that $z_{n}^{*}=z_{n}^{*} \chi_{\left[0, \delta_{n}\right)}$, and again using [KPS], Theorem II.3.1, we have also that

$$
\begin{aligned}
\left\|x+z_{n}\right\|_{\Lambda_{\phi}} & \geq \int_{0}^{\infty}\left|x^{*}-z_{n}^{*}\right|^{*} d \phi \\
& \geq \int_{0}^{\delta_{n}}\left(z_{n}^{*}-x^{*}\right) d \phi+\int_{\delta_{n}}^{\infty}\left(x^{*}-z_{n}^{*}\right) d \phi \\
& =\int_{0}^{\infty} z_{n}^{*} d \phi+\int_{0}^{\infty} x^{*} d \phi-2 \int_{0}^{\delta_{n}} x^{*} d \phi \\
& =\|x\|_{\Lambda_{\phi}}+\left\|z_{n}\right\|_{\Lambda_{\phi}}+o(1) .
\end{aligned}
$$

Hence

$$
\begin{aligned}
\left\|x+y_{n}\right\|_{\Lambda_{\phi}} & =\left\|x+z_{n}\right\|_{\Lambda_{\phi}}+o(1) \\
& =\|x\|_{\Lambda_{\phi}}+\left\|z_{n}\right\|_{\Lambda_{\phi}}+o(1) \\
& =\|x\|_{\Lambda_{\phi}}+\left\|y_{n}\right\|_{\Lambda_{\phi}}+o(1)
\end{aligned}
$$

Corollary 1.3. For any $\phi \in \Phi$, the Lorentz space $\left(\Lambda_{\phi},\|\cdot\|_{\Lambda_{\phi}}\right)$ has the Kadec-Klee property for convergence in measure.

As one application of Corollary 1.3, we give the following characterization of norm convergence in an arbitrary Lorentz space.

Corollary 1.4. If $\Lambda_{\phi}$ is an arbitrary Lorentz space on $[0, \alpha)$, and if $x \in \Lambda_{\phi},\left\{x_{n}\right\} \subseteq$ $\Lambda_{\phi}$, then the following statements are equivalent.

(i) $\left\|x_{n}-x\right\|_{\Lambda_{\phi}} \rightarrow 0$.

(ii) $\left\{x_{n}\right\}$ converges to $x$ in measure and for the weak topology $\sigma\left(\Lambda_{\phi}, \Lambda_{\phi}^{\prime}\right)$.

Proof. It is clear that only the implication (ii) $\Rightarrow$ (i) requires proof. We suppose first that $\lim _{t \rightarrow \infty} \phi(t)=\infty$, so that $\Lambda_{\phi}$ is separable. Assume that $\left\{x_{n}\right\} \subseteq \Lambda_{\phi}$ converges to $x \in \Lambda_{\phi}$ both weakly and in measure. It follows from [Fr], Corollary 29 and from Smulian's theorem ([Gr], Chapter 5, Corollary 3.1.2) there exists a subsequence $\left\{z_{n}\right\} \subseteq\left\{x_{n}\right\}$ and $z \in \Lambda_{\phi}$ such that $\left\{z_{n}^{*}\right\}$ converges to $z$ for the weak topology $\sigma\left(\Lambda_{\phi}, \Lambda_{\infty}\right)$. Let $0<\tau<t<\alpha$. Since $\left\{x_{n}\right\}$ converges to $x$ in measure, it follows that $\left\{z_{n}^{*}\right\}$ converges locally in measure to $x^{*}$, and so the sequence $\left\{z_{n}^{*}\right\}$ is uniformly bounded on $[\tau, t)$. It follows that

$$
\int_{\tau}^{t} z_{n}^{*} d m \rightarrow \int_{\tau}^{t} x^{*} d m
$$

so that the equality

$$
\int_{\tau}^{t} x^{*} d m=\int_{\tau}^{t} z d m
$$


holds for all $\tau, t \in(0, \alpha)$ with $\tau<t$. This implies that $x^{*}=z$, and since this argument applies equally to any subsequence of the sequence $\left\{x_{n}^{*}\right\}$, it follows that $\left\{x_{n}^{*}\right\}$ converges weakly to $x^{*}$. Since $\phi^{\prime} \in \Lambda_{\phi}^{\prime}$, it follows that

$$
\left\|x_{n}\right\|_{\Lambda_{\phi}}=\left\|x_{n}^{*}\right\|_{\Lambda_{\phi}}=\int_{0}^{\alpha} x_{n}^{*} \phi^{\prime} d m \rightarrow \int_{0}^{\alpha} x^{*} \phi^{\prime} d m=\left\|x^{*}\right\|_{\Lambda_{\phi}}=\|x\|_{\Lambda_{\phi}} .
$$

It now follows from Corollary 1.3 that $\left\|x_{n}-x\right\|_{\Lambda_{\phi}} \rightarrow 0$.

We now assume that $\lim _{t \rightarrow \infty} \phi(t)<\infty$. Although $\Lambda_{\phi}$ is not separable, observe that the space

$$
\Lambda_{\phi}(e):=\left\{f \in \Lambda_{\phi}: f=f \chi_{e}\right\}
$$

is separable for any measurable subset $e \subseteq[0, \infty)$ of finite measure. Let $x \in$ $\Lambda_{\phi},\left\{x_{n}\right\} \subseteq \Lambda_{\phi}$ and assume that $x_{n} \rightarrow x$ both in measure and for the weak topology $\sigma\left(\Lambda_{\phi}, \Lambda_{\phi}^{\prime}\right)$. If we set $y_{n}=x_{n}-x, n \geq 1$, it suffices to show that $\left\|y_{n}\right\|_{\Lambda_{\phi}} \rightarrow 0$. Passing to a subsequence if necessary, we may assume that

$$
m\left\{\left|y_{n}\right|>2^{-n}\right\}<2^{-n}, \quad n \geq 1 .
$$

It follows that

$$
\begin{aligned}
\left\|y_{n}\right\|_{\Lambda_{\phi}} & =\int_{[0, \infty)} y_{n}^{*}(t) d \phi(t) \\
& \leq \int_{\left[0,2^{-n}\right)} y_{n}^{*}(t) \phi^{\prime}(t) d t+\int_{\left[2^{-n}, \infty\right)} y_{n}^{*}(t) \phi^{\prime}(t) d t \\
& \leq \int_{\left[0,2^{-n}\right)} y_{n}^{*}(t) \phi^{\prime}(t) d t+2^{-n}\left(\phi(\infty)-\phi\left(2^{-n}\right)\right) .
\end{aligned}
$$

To complete the proof, it suffices to show that

$$
\int_{\left[0,2^{-n}\right)} y_{n}^{*}(t) \phi^{\prime}(t) d t \rightarrow 0
$$

For each $n \geq 1$, let $e_{n}$ be a measurable subset of $[0, \infty)$ for which

$$
\left(y_{n} \chi_{e_{n}}\right)^{*}=y_{n}^{*} \chi_{[0,2-n)} .
$$

It is clear that $y_{n} \chi_{e_{n}} \rightarrow 0$ in measure. We prove also that $y_{n} \chi_{e_{n}} \rightarrow 0$ for the weak topology $\sigma\left(\Lambda_{\phi}, \Lambda_{\phi}^{\prime}\right)$. Since $L_{\infty} \subseteq \Lambda_{\phi}$, it follows that $\Lambda_{\phi}^{\prime} \subseteq L_{1}$. For any $f \in \Lambda_{\phi}^{\prime} \subseteq L_{1}$, observe that

$$
\int_{[0, \infty)} y_{n} \chi_{e_{n}} f d m=\int_{[0, \infty)} y_{n} f d m-\int_{[0, \infty)} y_{n} \chi_{[0, \infty) \backslash e_{n}} f d m
$$

The first integral on the right-hand side tends to 0 by assumption, and since $f \in L_{1}$ and $\left|y_{n} \chi_{[0, \infty) \backslash e_{n}}\right| \leq 2^{-n}, \quad n \geq 1$, it follows also that the second integral on the right tends to 0 . Consequently, the sequence $\left\{y_{n} \chi_{e_{n}}\right\}$ converges to zero both in measure and for the weak topology $\sigma\left(\Lambda_{\phi}, \Lambda_{\phi}^{\prime}\right)$ and the desired conclusion that $\left\|y_{n}\right\|_{\Lambda_{\phi}} \rightarrow 0$ now follows from the first part of the proof applied to the space $\Lambda_{\phi}(e)$, where $e:=\bigcup_{n=1}^{\infty} e_{n}$.

In the case of $L_{1}$-spaces, it is well-known that "convergence in measure" can be replaced by "local convergence in measure" in the statement of assertion (ii) of the preceding Corollary 1.4. See, for example, [Gr], Proposition 4.1.2 of Chapter 5 . It is therefore of interest to note that this is not the case for general separable Lorentz spaces. In fact, suppose that the Lorentz function $\phi$ satisfies the condition 
$\lim _{t \rightarrow \infty} \phi(t) / t=0$ and set $x_{n}=\chi_{[n, n+1)}, n=1,2, \ldots$ It is not difficult to see that the sequence $\left\{x_{n}\right\}$ converges to 0 both weakly and locally in measure, but does not converge to 0 in $\Lambda_{\phi}$.

One further immediate consequence of Proposition 1.1 is that if the symmetric space $E$ on $[0, \alpha)$ has the Kadec-Klee property with respect to $\Lambda_{\infty}$, then $E$ is necessarily separable. The renorming theorem which follows is due to Sedaev [Se1], [Se2] . See also [DGL] .

Theorem 1.5. If $\left(E,\|\cdot\|_{E}\right)$ is a separable symmetric space on $[0, \alpha)$, then there exists an equivalent symmetric norm $\|\cdot\|_{0}$ on $E$ such that $\left(E,\|\cdot\|_{0}\right)$ has the KadecKlee property with respect to $\Lambda_{\infty}$.

Corollary 1.6. Let $\left(E,\|\cdot\|_{E}\right)$ be a separable symmetric space on $[0, \alpha)$ and let $x \in E,\left\{x_{n}\right\} \subseteq E$. If $\Gamma \subset E^{*}$ is any linear subspace for which $\Lambda_{\infty} \subseteq \Gamma$, then the following statements are equivalent.

(i) $\left\|x_{n}-x\right\|_{E} \rightarrow 0$.

(ii) $x_{n} \rightarrow x \quad \sigma(E, \Gamma)$ and $\left\|x_{n}^{*}-x^{*}\right\|_{E} \rightarrow 0$.

(iii) The sequence $\left\{x_{n}\right\}$ converges to $x$ in measure and $\left\|x_{n}^{*}-x^{*}\right\|_{E} \rightarrow 0$.

Proof. The implication (ii) $\Rightarrow$ (i) is a simple consequence of Theorem 1.5. The implication (i) $\Rightarrow$ (ii) follows from the rearrangement inequalities ([KPS], Theorem II 3.1)

$$
x_{n}^{*}-x^{*} \prec \prec\left(x_{n}-x\right)^{*}, \quad n=1,2, \ldots,
$$

and the symmetry of the norm on $E$. The implication (i) $\Rightarrow$ (iii) follows similarly. Finally, the implication (iii) $\Rightarrow$ (i) has been established in [CS1] .

Proposition 1.7. Let $\left(E,\|\cdot\|_{E}\right)$ be a separable symmetric space on $[0, \alpha)$. If $\left(E,\|\cdot\|_{E}\right)$ has the Kadec-Klee property with respect to $\Lambda_{\infty}$, then $\left(E,\|\cdot\|_{E}\right)$ has the Kadec-Klee property for convergence in measure.

Proof. We suppose that $x \in E,\left\{x_{n}\right\} \subseteq E$, that $x_{n} \rightarrow x$ in measure and that $\left\|x_{n}\right\|_{E} \rightarrow\|x\|_{E}$. Choose $y \in E^{\prime}=E^{*}$ such that $\|y\|_{E^{\prime}}=1$ and $\|x\|_{E}=\int_{0}^{\alpha} x(s) y(s) d s$. If $\phi(t)=\int_{0}^{t} y^{*}(s) d s, t>0$, then $\phi \in \Phi$ and for any $z \in E$,

$$
\|z\|_{\Lambda_{\phi}}=\int_{0}^{\alpha} z^{*}(s) y^{*}(s) d s \leq\left\|z^{*}\right\|_{E}\left\|y^{*}\right\|_{E^{\prime}}=\|z\|_{E} .
$$

Since

$$
\|x\|_{E}=\int_{0}^{\alpha} x(s) y(s) d s \leq \int_{0}^{\alpha} x^{*}(s) y^{*}(s) d s=\|x\|_{\Lambda_{\phi}},
$$

it follows that $\|x\|_{E}=\|x\|_{\Lambda_{\phi}}$. Further

$$
\limsup \left\|x_{n}\right\|_{\Lambda_{\phi}} \leq \limsup \left\|x_{n}\right\|_{E}=\|x\|_{E}=\|x\|_{\Lambda_{\phi}} .
$$

On the other hand, by Proposition 1.2, it follows that

$$
\liminf \left\|x_{n}\right\|_{\Lambda_{\phi}}=\liminf \left(\|x\|_{\Lambda_{\phi}}+\left\|x_{n}-x\right\|_{\Lambda_{\phi}}+o(1)\right) \geq\|x\|_{\Lambda_{\phi}} .
$$

Consequently, $\left\|x_{n}\right\|_{\Lambda_{\phi}} \rightarrow\|x\|_{\Lambda_{\phi}}$ and Corollary 1.3 now implies that $\left\|x_{n}-x\right\|_{\Lambda_{\phi}} \rightarrow 0$. Via the embedding $\Lambda_{\infty} \subseteq \Lambda_{\phi}$, it follows that $\left\{x_{n}\right\}$ converges to $x$ pointwise on $\Lambda_{\infty}$. The stated assumption on $E$ now immediately implies that $\left\|x_{n}-x\right\|_{E} \rightarrow 0$ and this completes the proof of the proposition. 
Lemma 1.8. Let $\left(E,\|\cdot\|_{E}\right)$ be a separable symmetric space on $[0, \alpha)$. If $x, x_{n} \in$ $E, n \geq 1$ and if $x_{n} \rightarrow x$ locally in measure, then there exists a sequence $\left\{e_{n}\right\}$ of measurable subsets of finite measure such that $\chi_{e_{n}} \rightarrow 1$ locally in measure, and a subsequence $\left\{y_{n}\right\} \subseteq\left\{x_{n}\right\}$ such that

$$
\left\|y_{n} \chi_{e_{n}}-x\right\|_{E} \rightarrow 0, \quad\left\|x\left(1-\chi_{e_{n}}\right)\right\|_{E} \rightarrow 0 .
$$

The preceding lemma follows immediately from [DDDLS], Proposition 2.1.

\section{Strictly $K$-MONOTONE SYMmetric NORMS}

In this section, we characterize the Kadec-Klee property with respect to $\Lambda_{\infty}$ in terms of the Kadec-Klee property for convergence in measure (respectively, local convergence in measure) and certain key properties that link the $K$-functional to the metric geometry. We first make the following basic definitions. Let $\left(E,\|\cdot\|_{E}\right)$ be a symmetric space on $([0, \alpha), m)$. The norm $\|\cdot\|_{E}$ on $E$ is said to be strictly $K$-monotone if and only if

$$
x, y \in E, x \prec \prec y, x^{*} \neq y^{*} \Rightarrow\|x\|_{E}<\|y\|_{E} .
$$

The norm $\|\cdot\|_{E}$ on $E$ is said to be locally uniformly strictly K-monotone if and only if

$$
x \in E,\left\{x_{n}\right\} \subseteq E, \quad x \prec \prec x_{n}, n=1,2, \ldots,\left\|x_{n}\right\|_{E} \rightarrow\|x\|_{E} \Rightarrow\left\|x_{n}^{*}-x^{*}\right\|_{E} \rightarrow 0 .
$$

Proposition 2.1. Let $\left(E,\|\cdot\|_{E}\right)$ be a separable symmetric space on $[0, \alpha)$. If $\left(E,\|\cdot\|_{E}\right)$ has the Kadec-Klee property, then the norm on $E$ is strictly $K$-monotone.

Proof. We suppose that $x, y \in E$, that $x=x^{*}, y=y^{*}, x \prec \prec y$ and that $x \neq y$. If

$$
\phi(t)=\int_{0}^{t} x(s) d s, \quad \psi(t)=\int_{0}^{t} y(s) d s, \quad t>0,
$$

then $\phi, \psi \in \Phi, \phi(t) \leq \psi(t), t>0$ and there exist $\tau>0, \epsilon>0$ such that $\phi(\tau)+\epsilon<$ $\psi(\tau)$. Let $\widehat{\phi}$ be the smallest member of $\Phi$ for which $\phi \leq \widehat{\phi}$ and $\phi(\tau)+\epsilon=\widehat{\phi}(\tau)$. The graph of the function $\widehat{\phi}$ is the boundary of the convex hull of the set

$$
\{(t, z): z \leq \phi(t)\} \cup\left\{(t, z): z \leq \min \left(\phi(\tau)+\epsilon, \frac{\phi(\tau)+\epsilon}{\tau} t\right)\right\} .
$$

For some $\beta>0$, the function $\widehat{\phi}$ is affine on the intervals $(\tau-\beta, \tau)$ and $(\tau, \tau+\beta)$; in addition, the slope of the function $\widehat{\phi}$ on the first interval is strictly greater than that on the second interval. The function $p(t)=\widehat{\phi}^{\prime}(t)$ is constant on the same intervals and $p(\tau-0)>p(\tau+0)$. Let $\delta=2^{-1}(p(\tau-0)-p(\tau+0)), I_{\beta}=(\tau-\beta, \tau+\beta)$,

$$
q(t)= \begin{cases}p(t), & \text { if } t \notin I_{\beta} ; \\ p(\tau+0)+\delta, & \text { if } t \in I_{\beta},\end{cases}
$$

and let

$$
\bar{\phi}_{\beta}(t)=\int_{0}^{t} q(s) d s, \quad t \geq 0 .
$$

By construction, $q=q^{*}, \bar{\phi}_{\beta}(t) \in \Phi, \bar{\phi}_{\beta}(t)=\widehat{\phi}(t)$ for $t \notin I_{\beta}$ and $\bar{\phi}_{\beta}(t)<\widehat{\phi}(t)$ for $t \in I_{\beta}$. It is clear also that if $\beta \rightarrow 0$, then $\bar{\phi}_{\beta}(t) \rightarrow \widehat{\phi}(t)$ for all $t \geq 0$. Consequently, 
there exists $\beta>0$ such that the inequality $\phi(t) \leq \bar{\phi}_{\beta}(t)$ holds for all $t>0$, and with this choice of $\beta$ it follows that $x \prec \prec q \prec \prec p \prec \prec y$. Let

$$
z_{n}(t)= \begin{cases}\operatorname{sgn}\left(\sin \left[2^{n}\left(\frac{\pi}{\beta}(t-\tau+\beta)\right)\right]\right), & t \in I_{\beta} ; \\ 0, & \text { otherwise }\end{cases}
$$

be the Rademacher functions transferred to $I_{\beta}$, and let $q_{n}=q+\delta z_{n}, n=1,2, \ldots$. It is easy to see that $q_{n}^{*}=p$ and, therefore, $x \prec \prec q \prec \prec q_{n} \prec \prec y, n=1,2, \ldots$ Since $\left\{z_{n}\right\}$ converges to 0 weakly, it follows that $\left\{q_{n}\right\}$ converges to $q$ weakly. If $\|x\|_{E}=\|y\|_{E}$, then $\left\|q_{n}\right\|_{E}=\|q\|_{E}$ for all $n=1,2, \ldots$ and so, since $E$ has the KadecKlee property, it follows that $\left\|q_{n}-q\right\|_{E} \rightarrow 0$. This is impossible, since $\left\|q_{n}-q\right\|_{E}=$ $\delta\left\|\chi_{I_{\beta}}\right\|_{E}>0$, and this suffices to complete the proof of the proposition.

We remark that it will be shown in Theorem 2.11 below that for separable Lorentz spaces, the Kadec-Klee property is in fact equivalent to strict $K$-monotonicity of the norm. Before proceeding, we need some additional notation. Let $E$ be a separable symmetric space on $([0, \alpha), m)$. If $x=x^{*} \in E$, and if $\epsilon, \tau>0$, we denote by $W(x, \tau, \epsilon)$ the set of all $y \in E$ for which

$$
y=y^{*}, x \prec \prec y \quad \text { and } \quad \int_{0}^{\tau} x(s) d s+\epsilon \leq \int_{0}^{\tau} y(s) d s .
$$

Corollary 2.2. There exists $z \in W(x, \tau, \epsilon)$ such that $z \prec \prec y$ for all $y \in W(x, \tau, \epsilon)$.

Proof. We define $\psi$ to be the function $\widehat{\phi}$ constructed in the previous proof. Let $z=\psi^{\prime}$.

We will frequently use the following simple fact.

Lemma 2.3. In a separated topological vector space, a sequence $\left\{x_{n}\right\}$ converges to $x$ if and only if each subsequence $\left\{y_{n}\right\} \subseteq\left\{x_{n}\right\}$ contains a subsequence $\left\{z_{n}\right\}$ converging to $x$.

Proposition 2.4. Let $\left(E,\|\cdot\|_{E}\right)$ be a separable symmetric space on $([0, \alpha), m)$ with strictly $K$-monotone norm. If $x \in E,\left\{x_{n}\right\} \subseteq E$, if $x \prec \prec x_{n}, n=1,2, \ldots$ and if $\left\|x_{n}\right\|_{E} \rightarrow\|x\|_{E}$, then the sequence $\left\{x_{n}^{*}\right\}$ converges to $x^{*}$ in measure.

Proof. Suppose that $x \in E,\left\{x_{n}\right\} \subseteq E$, that $x \prec \prec x_{n}, n=1,2, \ldots$, and $\left\|x_{n}\right\|_{E} \rightarrow$ $\|x\|_{E}$. Since $x^{*}(\infty)=0$, it suffices to show that $x_{n}^{*} \rightarrow x^{*}$ almost everywhere. If $C=\sup \left\|x_{n}\right\|_{E}<\infty$, the inequalities $x_{n}^{*}(s) \chi_{[0, s)} \leq x_{n}^{*}, s \in(0, \alpha)$, imply the uniform estimate

$$
x_{n}^{*}(s) \leq C\left\|\chi_{[0, s)}\right\|_{E}^{-1}, \quad s \in(0, \alpha), \quad n=1,2, \ldots
$$

Using Helly's principle and passing to a subsequence and relabelling if necessary, we may assume that the sequence $\left\{x_{n}^{*}\right\}$ converges to some non-increasing function $z$ almost everywhere on $[0, \alpha)$. Via Lemma 2.3 , it suffices to show that $z=x^{*}$. Since

$$
\int_{\beta}^{t} x_{n}^{*}(s) d s \rightarrow \int_{\beta}^{t} z(s) d s
$$

for all $t>\beta>0$, it suffices further to show that

$$
\int_{0}^{t} x_{n}^{*}(s) d s \rightarrow \int_{0}^{t} x^{*}(s) d s, \quad t>0 .
$$


If this is not so, then passing to a subsequence and relabelling if necessary, there exist $\tau \in(0, \alpha), \epsilon>0$ such that

$$
\int_{0}^{\tau} x^{*}(s) d s+\epsilon \leq \int_{0}^{\tau} x_{n}^{*}(s) d s, \quad n=1,2, \ldots
$$

By Corollary 2.2, there exists $y=y^{*}$ with $x \prec \prec y \prec \prec x_{n}^{*}, n=1,2, \ldots$ such that

$$
\int_{0}^{\tau} x^{*}(s) d s+\epsilon \leq \int_{0}^{\tau} y^{*}(s) d s
$$

The last inequality implies in particular that $y \neq x$, and so strict $K$-monotonicity of the norm on $E$ implies that

$$
\|x\|_{E}<\|y\|_{E} \leq\left\|x_{n}\right\|_{E}, \quad n=1,2, \ldots
$$

and this contradicts the assumption that $\left\|x_{n}\right\|_{E} \rightarrow\|x\|_{E}$. This completes the proof of the proposition.

Lemma 2.5. Let $\left(E,\|\cdot\|_{E}\right)$ be a separable symmetric space on $([0, \alpha), m)$. If $x \in$ $E,\left\{x_{n}\right\} \subseteq E$ and if $x_{n} \rightarrow x \sigma\left(E, \Lambda_{\infty}\right)$, then there exists a subsequence $\left\{z_{n}\right\} \subseteq\left\{x_{n}\right\}$ and a sequence $\left\{y_{n}\right\} \subseteq E$ such that $\left\|y_{n}\right\|_{E} \rightarrow 0$ and

$$
x^{*} \prec \prec z_{n}^{*}+y_{n}^{*}, \quad n=1,2, \ldots
$$

Proof. Multiplying by a unimodular function if necessary, we may assume that $x \geq 0$. We set

$$
M_{n}=\left\{n^{-1}<x \leq n\right\}, \quad M_{n}^{c}=[0, \alpha) \backslash M_{n}, \quad n=1,2, \ldots .
$$

Since $E$ is separable, we have $m\left(M_{n}\right)<\infty$, and hence $\chi_{M_{n}} \in E$ for all $n=1,2, \ldots$. We consider only those $n$ for which $\beta_{n}=\left\|\chi_{M_{n}}\right\|_{E} \neq 0$. Set

$$
\gamma_{n}=2^{-n} \beta_{n}^{-1}\left(n-n^{-1}\right), \quad k(n)=\left[2^{n} \beta_{n}\right],
$$

where [.] denotes the usual integer part function. For each $n=1,2, \ldots$, let

$$
a_{i}=n^{-1}+i \gamma_{n}, \quad i=0,1, \ldots, k(n), \quad a_{k(n)+1}=n,
$$

and set $e_{i}^{n}=\left\{a_{i} \leq x<a_{i+1}\right\}, i=0,1, \ldots, k(n)$. It is clear that $M_{n}=\bigcup_{i=0}^{k(n)} e_{i}^{n}$. We consider the averaging operators

$$
P_{n} y=\sum_{i=0}^{k(n)}\left(\frac{1}{m\left(e_{i}^{n}\right)} \int_{e_{i}^{n}} y d m\right) \chi_{e_{i}^{n}}, \quad y \in \Lambda_{1}
$$

Since

$$
\left|x-P_{n} x\right| \leq\left|x \chi_{M_{n}^{c}}\right|+\gamma_{n} \chi_{M_{n}}
$$

it follows from the order continuity of the norm on $E$ that

$$
\left\|x-P_{n} x\right\|_{E} \leq\left\|x \chi_{M_{n}^{c}}\right\|_{E}+2^{-n}\left(n-n^{-1}\right) \rightarrow 0 .
$$

Clearly, since $x_{k} \rightarrow x$ w.r.t. $\sigma\left(E, \Lambda_{\infty}\right),\left\|P_{n} x_{k}-P_{n} x\right\|_{E} \rightarrow 0$ as $k \rightarrow \infty$ for each fixed $n$. Using the fact that $\left\|P_{n} x-x\right\|_{E} \rightarrow 0$, it follows that there exists a subsequence $\left\{z_{n}\right\} \subseteq\left\{x_{n}\right\}$ such that $\left\|P_{n} z_{n}-x\right\|_{E} \rightarrow 0$. If we set

$$
y_{n}=\left(x-P_{n} z_{n}\right)^{*}, \quad n=1,2, \ldots,
$$

then $\left\|y_{n}\right\|_{E} \rightarrow 0$. Since $P_{n} z \prec \prec z$ for every $z \in \Lambda_{1}$ ([KPS], Chapter II, (3.11)), we obtain that

$$
x^{*} \prec \prec\left(P_{n} z_{n}\right)^{*}+\left(x-P_{n} z_{n}\right)^{*} \prec \prec z_{n}^{*}+y_{n}^{*}, \quad n=1,2, \ldots,
$$


and this suffices to establish the assertion of the lemma.

Lemma 2.6. Let $\left(E,\|\cdot\|_{E}\right)$ be a separable symmetric space on $([0, \alpha), m)$ with strictly K-monotone norm. If $x \in E,\left\{x_{n}\right\} \subseteq E$, if $x_{n} \rightarrow x \sigma\left(E, \Lambda_{\infty}\right)$ and if $\left\|x_{n}\right\|_{E} \rightarrow\|x\|_{E}$, then the sequence $\left\{x_{n}^{*}\right\}$ converges to $x^{*}$ in measure.

Proof. By Lemma 2.5, there exists a subsequence $\left\{z_{n}\right\} \subseteq\left\{x_{n}\right\}$ and a sequence $\left\{y_{n}\right\} \subseteq E$ such that

$$
\left\|y_{n}\right\|_{E} \rightarrow 0 \text { and } x^{*} \prec \prec z_{n}^{*}+y_{n}^{*}, \quad n=1,2, \ldots
$$

Since $\left\|x_{n}\right\|_{E} \rightarrow\|x\|_{E}$, it follows also that $\left\|z_{n}^{*}+y_{n}^{*}\right\|_{E} \rightarrow\|x\|_{E}$. Consequently, Proposition 2.4 implies that the sequence $\left\{z_{n}^{*}+y_{n}^{*}\right\}$ converges to $x^{*}$ in measure. Since $\left\|y_{n}\right\|_{E} \rightarrow 0$, it follows also that $\left\{z_{n}^{*}\right\}$ converges to $x^{*}$ in measure. Since the preceding argument applies equally to any subsequence of the sequence $\left\{x_{n}\right\}$, it now follows that $\left\{x_{n}^{*}\right\}$ converges to $x^{*}$ in measure.

We now state the principal result of this section.

Theorem 2.7. If $\left(E,\|\cdot\|_{E}\right)$ is a separable symmetric space on $([0, \alpha), m)$, then the following statements are equivalent.

(i) $\left(E,\|\cdot\|_{E}\right)$ has the Kadec-Klee property with respect to $\Lambda_{\infty}$.

(ii) The norm $\|\cdot\|_{E}$ on $E$ is strictly $K$-monotone and $\left(E,\|\cdot\|_{E}\right)$ has the Kadec-Klee property for convergence in measure.

(iii) The norm $\|\cdot\|_{E}$ on $E$ is strictly $K$-monotone and $\left(E,\|\cdot\|_{E}\right)$ has the Kadec-Klee property for local convergence in measure.

(iv) The norm $\|\cdot\|_{E}$ on $E$ is locally uniformly strictly $K$-monotone.

Proof. We prove first the equivalences (i) $\Longleftrightarrow$ (ii) $\Longleftrightarrow$ (iv). The implication (i) $\Rightarrow$ (ii) is an immediate consequence of Propositions 1.7, 2.1 while the implication (ii) $\Rightarrow$ (iv) follows from Proposition 2.4. To show the implication (iv) $\Rightarrow$ (i), let $x \in E,\left\{x_{n}\right\} \subseteq E$ and assume that $x_{n} \rightarrow x \sigma\left(E, \Lambda_{\infty}\right)$ and $\left\|x_{n}\right\|_{E} \rightarrow\|x\|_{E}$. By Lemma 2.5, there exists a subsequence $\left\{z_{n}\right\} \subseteq\left\{x_{n}\right\}$ and a sequence $\left\{y_{n}\right\} \subseteq E$ such that

$$
\left\|y_{n}\right\|_{E} \rightarrow 0 \text { and } x^{*} \prec \prec z_{n}^{*}+y_{n}^{*}, \quad n=1,2, \ldots
$$

Since $\left\|x_{n}\right\|_{E} \rightarrow\|x\|_{E}$, it follows also that $\left\|z_{n}^{*}+y_{n}^{*}\right\|_{E} \rightarrow\|x\|_{E}$. Since the norm on $E$ is locally uniformly strictly $K$-monotone, it follows that $\left\|z_{n}^{*}+y_{n}^{*}-x^{*}\right\|_{E} \rightarrow 0$ and so also $\left\|z_{n}^{*}-x^{*}\right\|_{E} \rightarrow 0$. Since the preceding argument applies equally to any subsequence of the sequence $\left\{x_{n}\right\}$, Lemma 2.3 now implies that $\left\|x_{n}^{*}-x^{*}\right\|_{E} \rightarrow 0$. That $\left\|x_{n}-x\right\|_{E} \rightarrow 0$ is now a consequence of Corollary 1.6.

The implication (iii) $\Rightarrow$ (ii) is trivial.

To show the implication (ii) $\Rightarrow$ (iii), it is clear that we may take $\alpha$ to be $\infty$, otherwise there is nothing to prove. Let $x \in E,\left\{x_{n}\right\} \subseteq E$ and assume that $x_{n} \rightarrow x$ locally in measure and that $\left\|x_{n}\right\|_{E} \rightarrow\|x\|_{E}=1$. It suffices to prove that the sequence $\left\{x_{n}\right\}$ contains some subsequence converging in $E$ to $x$. By passing to a subsequence and relabelling if necessary, it follows from Lemma 1.8 that there exist measurable subsets $e_{n} \subseteq[0, \infty), n \geq 1$, of finite measure such that $\chi_{e_{n}} \rightarrow 1$ locally in measure and

$$
\left\|x_{n} \chi_{e_{n}}-x\right\|_{E} \rightarrow 0, \quad\left\|x \chi_{f_{n}}\right\|_{E} \rightarrow 0
$$

where $f_{n}=[0, \infty) \backslash e_{n}, n \geq 1$. If we set $y_{n}=x_{n} \chi_{f_{n}}$ for all $n \geq 1$, then it suffices to show that $\left\|y_{n}\right\|_{E} \rightarrow 0$. If this is not so, then it follows that $\left\{y_{n}\right\}$ does not 
converge to zero in measure, since $E$ has the Kadec-Klee property for convergence in measure, by condition (ii), and $x_{n} \chi_{e_{n}} \rightarrow x$ in measure. Consequently, passing to subsequences, there exists a positive number $\epsilon>0$ and measurable subsets $f_{n}^{\prime} \subseteq f_{n}, n \geq 1$, such that $m\left(f_{n}^{\prime}\right)=\epsilon$ and $\left|y_{n}\right| \chi_{f_{n}^{\prime}} \geq \epsilon \chi_{f_{n}^{\prime}}, n \geq 1$. If we set

$$
v_{n}=x_{n} \chi_{e_{n}}+\epsilon \chi_{f_{n}^{\prime}}, \quad n \geq 1
$$

then $\left|v_{n}\right| \leq\left|x_{n}\right|$ for all $n \geq 1$ and $v_{n} \rightarrow x$ locally in measure. By lower-semicontinuity of the norm on $E$ for local convergence in measure, it follows that $\left\|v_{n}\right\|_{E} \rightarrow\|x\|_{E}$. We now set

$$
w_{n}=x \chi_{e_{n}}+\epsilon \chi_{f_{n}^{\prime}}, \quad n \geq 1
$$

Since $\left\|w_{n}-v_{n}\right\|_{E} \rightarrow 0$, it follows also that $\left\|w_{n}\right\|_{E} \rightarrow\|x\|_{E}$. Since the sequence $\left\{w_{n}\right\}$ is $\sigma\left(E, \Lambda_{\infty}\right)$-convergent to $x$, the implication (ii) $\Rightarrow(\mathrm{i})$ shows that $\left\|w_{n}-x\right\|_{E} \rightarrow 0$. Consequently,

$$
\epsilon\left\|\chi_{f_{n}^{\prime}}\right\|_{E} \leq\left\|w_{n}-x\right\|_{E}+\left\|x-x \chi_{e_{n}}\right\|_{E} \rightarrow 0 .
$$

Since $\left(\chi_{f_{n}^{\prime}}\right)^{*}=\chi_{[0, \epsilon)}, n=1,2, \ldots$, this yields a contradiction and the proof of the theorem is complete.

We remark immediately that if $\phi \in \Phi$ is strictly concave with $\phi(\infty)<\infty$, then the Lorentz space $\left(\Lambda_{\phi},\|\cdot\|_{\phi}\right)$ has strictly $K$-monotone norm (see Theorem 2.11 below), has the Kadec-Klee property for convergence in measure, by Corollary 1.3, and so satisfies the assertion of statement (ii) of the preceding Theorem 2.7. However, since the norm in this case is not order-continuous, it follows that $\left(\Lambda_{\phi}, \|\right.$. $\left.\|_{\phi}\right)$ does not have the Kadec-Klee property with respect to $\Lambda_{\infty}$. Consequently, the implication (ii) $\Rightarrow$ (i) does not remain valid if the assumption that $E$ be separable is omitted. We observe next that the two properties given in statement (ii) of Theorem 2.7 above are in fact independent. The space $L_{1}[0,1]$ with its usual norm has the Kadec-Klee property for convergence in measure, but it is easily seen that the usual norm on $L_{1}[0,1]$ is not strictly $K$-monotone. We now present an example of a separable symmetric space on $([0,1), m)$ with strictly $K$-monotone norm but which does not have the Kadec-Klee property for convergence in measure.

Example 2.8. Let $\delta>0$ and let $\phi_{1}, \phi_{2}$ be strictly concave functions from $\Phi$ such that $\phi_{2}(1)>\phi_{1}(1)+\delta$ and $\lim _{t \rightarrow 0} \frac{\phi_{2}(t)}{\phi_{1}(t)}=0$. We set $E=\Lambda_{\phi_{1}} \cap \Lambda_{\phi_{2}}$ with norm given by $\|\cdot\|_{E}=\max \left\{\|\cdot\|_{\Lambda_{\phi_{1}}},\|\cdot\|_{\Lambda_{\phi_{2}}}\right\}$. It follows from the strict concavity of $\phi_{1}, \phi_{2}$ that the norm on $E$ is strictly $K$-monotone. To show that $\left(E,\|\cdot\|_{E}\right)$ does not have the Kadec-Klee property for convergence in measure, we set $x=\chi_{[0,1]}$ and define

$$
x_{n}=x+\frac{\delta}{\phi_{1}\left(\frac{1}{n}\right)} \chi_{\left[0, \frac{1}{n}\right)}, \quad n=1,2, \ldots
$$

It is clear that the sequence $\left\{x_{n}\right\}$ converges to $x$ in measure and

$$
\lim _{n \rightarrow \infty}\left\|x_{n}\right\|_{E}=\lim _{n \rightarrow \infty}\left(\phi_{2}(1)+\frac{\delta \phi_{2}\left(\frac{1}{n}\right)}{\phi_{1}\left(\frac{1}{n}\right)}\right)=\phi_{2}(1)=\|x\|_{E} .
$$

However, since $\left\|x_{n}-x\right\|_{E}=\delta>0$ for $n>1$, it follows that $\left(E,\|\cdot\|_{E}\right)$ does not have the Kadec-Klee property for convergence in measure.

By combining Theorem 1.5 and Theorem 2.7, we obtain immediately the following consequence. 
Theorem 2.9. If $\left(E,\|\cdot\|_{E}\right)$ is a separable symmetric space on $([0, \infty), m)$, then there exists an equivalent norm $\|\cdot\|_{0}$ on $E$ such that $\left(E,\|\cdot\|_{0}\right)$ is a symmetric space with the Kadec-Klee property for local convergence in measure.

We mention finally that the implication (ii) $\Rightarrow$ (iii) of Theorem 2.7 may fail if the norm on the symmetric space $E$ is not strictly $K$-monotone. By way of example, we consider the symmetric space $E$ which is the $\|\cdot\|_{\Lambda_{1}}$-closure of $\Lambda_{\infty}$ in $\Lambda_{1}$. It is clear that $E$ is separable, and Corollary 1.3 implies that $E$ has the Kadec-Klee property for convergence in measure. To see that $E$ does not have the KadecKlee property for local convergence in measure, we let $x=\chi_{[0,1)}$, and set $x_{n}=$ $x+\chi_{[n, n+1)}, n=1,2, \ldots$ It is clear that the sequence $\left\{x_{n}\right\}$ converges locally in measure to $x$. Further,

$$
\|x\|_{E}=\int_{0}^{1} x^{*} d m=1=\int_{0}^{1} x_{n}^{*} d m=\left\|x_{n}\right\|_{E}, \quad n=1,2, \ldots
$$

However, since

$$
\left(x_{n}-x\right)^{*}=\chi_{[0,1)}, \quad n=1,2, \ldots
$$

it follows that $\left\{x_{n}\right\}$ is not $\|\cdot\|_{E}$-convergent to $x$ and so $E$ fails to have the KadecKlee property for local convergence in measure. Of course, in this example it is easy to see that the space $E$ fails to have strictly $K$-monotone norm.

We now characterize the Kadec-Klee property in separable Lorentz spaces in terms of the rearrangement-invariant structure.

Theorem 2.10. Let $\Lambda_{\phi}$ be a separable Lorentz space on $[0, \alpha)$. If $\|\cdot\|$ is a symmetric norm on $\Lambda_{\phi}$ that is equivalent to the norm $\|\cdot\|_{\Lambda_{\phi}}$, then the following statements are equivalent.

(i) The norm $\|\cdot\|$ on $\Lambda_{\phi}$ is strictly $K$-monotone.

(ii) $\left(\Lambda_{\phi},\|\cdot\|\right)$ has the Kadec-Klee property.

Proof. It suffices via Proposition 2.1, to show that strict $K$-monotonicity of the norm $\|\cdot\|$ on $E$ implies that $E$ has the Kadec-Klee property. Assume then that the norm $\|\cdot\|$ on $\Lambda_{\phi}$ is strictly $K$-monotone and suppose that $\left\{x_{n}\right\} \subseteq \Lambda_{\phi}$ is $\sigma\left(\Lambda_{\phi}, \Lambda_{\phi}^{\prime}\right)$ convergent to $x \in \Lambda_{\phi}$ and $\left\|x_{n}\right\| \rightarrow\|x\|$. From Lemma 2.6, it follows that $\left\{x_{n}^{*}\right\}$ converges to $x^{*}$ in measure. The same argument as given in the proof of Corollary 1.4 shows that $\left\|x_{n}^{*}\right\|_{\Lambda_{\phi}} \rightarrow\left\|x^{*}\right\|_{\Lambda_{\phi}}$ and so it follows from Corollary 1.3 that $\left\|x_{n}^{*}-x^{*}\right\|_{\Lambda_{\phi}} \rightarrow 0$. The assertion of the theorem now follows from Corollary 1.6.

We remark that the Kadec-Klee property does not coincide with the Kadec-Klee property with respect to $\Lambda_{\infty}$, even in the class of separable symmetric spaces with strictly $K$-monotone norm. Indeed, it is not possible to replace assertion (ii) in the preceding theorem by the assertion that $\left(\Lambda_{\phi},\|\cdot\|\right)$ has the Kadec-Klee property with respect to $\Lambda_{\infty}$. In fact, the space $E$ of Example 2.8 coincides as a set with the Lorentz space $\Lambda_{\phi_{1}}$, the norms $\|\cdot\|_{E}$ and $\|\cdot\|_{\Lambda_{\phi_{1}}}$ are equivalent and the norm $\|\cdot\|_{E}$ is strictly $K$-monotone. It follows from Theorem 2.10 that $\left(E,\|\cdot\|_{E}\right)$ has the Kadec-Klee property. As seen above, the space $\left(E,\|\cdot\|_{E}\right)$ does not have the Kadec-Klee property for convergence in measure, and so, by Theorem 2.7, the space $\left(E,\|\cdot\|_{E}\right)$ does not have the Kadec-Klee property with respect to $\Lambda_{\infty}$. In contrast, we now show for separable Lorentz spaces $\left(\Lambda_{\phi},\|\cdot\|_{\Lambda_{\phi}}\right)$, that strict $K$-monotonicity 
of the given norm $\|\cdot\|_{\Lambda_{\phi}}$ is not only equivalent to the Kadec-Klee property, but also to the Kadec-Klee property with respect to $\Lambda_{\infty}$.

Theorem 2.11. If $\left(\Lambda_{\phi},\|\cdot\|_{\Lambda_{\phi}}\right)$ is a separable Lorentz space on $([0, \alpha), m)$, then the following statements are equivalent.

(i) $\phi$ is strictly concave.

(ii) The norm $\|\cdot\|_{\Lambda_{\phi}}$ is strictly $K$-monotone.

(iii) The norm $\|\cdot\|_{\Lambda_{\phi}}$ is locally uniformly strictly $K$-monotone.

(iv) $\left(\Lambda_{\phi},\|\cdot\|_{\Lambda_{\phi}}\right)$ has the Kadec-Klee property with respect to $\Lambda_{\infty}$.

(v) $\left(\Lambda_{\phi},\|\cdot\|_{\Lambda_{\phi}}\right)$ has the Kadec-Klee property.

Proof. The equivalences (ii) $\Longleftrightarrow$ (iii) $\Longleftrightarrow$ (iv) follow from Theorem 2.7 and Corollary 1.3. The equivalence (ii) $\Longleftrightarrow(\mathrm{v})$ is a special case of Theorem 2.10. The equivalence (i) $\Longleftrightarrow$ (iv) has been proved by Sedaev [Se1] in the case that $\alpha<\infty$. It follows immediately that the equivalence (i) $\Longleftrightarrow$ (ii) also holds if $\alpha<\infty$. If the norm $\|\cdot\|_{\Lambda_{\phi}}$ is strictly $K$-monotone, it now follows that the restriction of $\phi$ to every finite interval is strictly concave and this suffices to establish the implication (ii) $\Rightarrow$ (i). We show now the implication (i) $\Rightarrow$ (ii).

Let $x=x^{*}, y=y^{*} \in \Lambda_{\phi}$ and suppose that $x \prec \prec y$, and that $x^{*} \neq y^{*}$. We set

$$
u(t)=\int_{[0, t)} x^{*}(s) d s, \quad v(t)=\int_{[0, t)} y^{*}(s) d s, \quad t>0 .
$$

It is clear that $0 \leq u \leq v$ and that there exists $t_{0} \in[0, \alpha)$ such that $u\left(t_{0}\right)<v\left(t_{0}\right)$. Since $u$ and $v$ are continuous, there exists $t_{1} \in\left[t_{0}, \alpha\right)$ so that $v(t)-u(t) \geq \epsilon$ for all $t \in\left[t_{0}, t_{1}\right]$, for some $\epsilon>0$. Also, since $\phi$ is strictly concave, $-\phi^{\prime}$ is strictly increasing. Via integration by parts, for all $b \in\left(t_{1}, \alpha\right)$,

$$
\begin{aligned}
\int_{[0, b)} x^{*}(s) \phi^{\prime}(s) d s & =\lim _{a \rightarrow 0+}\left(u(b) \phi^{\prime}(b-)-u(a) \phi^{\prime}(a+)+\int_{(a, b)} u(t) d\left(-\phi^{\prime}\right)(t)\right) \\
& =u(b) \phi^{\prime}(b-)+\int_{[0, b)} u(t) d\left(-\phi^{\prime}\right)(t),
\end{aligned}
$$

because $u(a) \phi^{\prime}(a+) \rightarrow 0$ as $a \rightarrow 0+$. So,

$$
\begin{aligned}
\int_{[0, b)} x^{*}(s) \phi^{\prime}(s) d s & =u(b) \phi^{\prime}(b-)+\left(\int_{\left[0, t_{0}\right]}+\int_{\left(t_{0}, t_{1}\right)}+\int_{\left[t_{1}, b\right)}\right) u(t) d\left(-\phi^{\prime}\right)(t) \\
& \leq v(b) \phi^{\prime}(b-)+\left(\int_{\left[0, t_{0}\right]}+\int_{\left(t_{0}, t_{1}\right)}+\int_{\left[t_{1}, b\right)}\right) v(t) d\left(-\phi^{\prime}\right)(t) \\
& -\int_{\left(t_{0}, t_{1}\right)}(v(t)-u(t)) d\left(-\phi^{\prime}\right)(t) \\
& \leq v(b) \phi^{\prime}(b-)+\int_{[0, b)} v(t) d\left(-\phi^{\prime}\right)(t)-\epsilon\left(-\phi^{\prime}\left(t_{1}-\right)+\phi^{\prime}\left(t_{0}+\right)\right) \\
& =\int_{[0, b)} y^{*}(s) \phi^{\prime}(s) d s-\epsilon\left(\phi^{\prime}\left(t_{0}+\right)-\phi^{\prime}\left(t_{1}-\right)\right) .
\end{aligned}
$$

Letting $b \rightarrow \alpha$, we see that $\|x\|_{\Lambda_{\phi}}<\|y\|_{\Lambda_{\phi}}$. 
This suffices to complete the proof of the implication (i) $\Rightarrow$ (ii) .

As noted after the proof of Theorem 2.7, the above proof (which is due to the referee) also establishes (i) $\Rightarrow$ (ii) in the nonseparable case. We remark that the equivalences (ii) $\Longleftrightarrow$ (iv) $\Longleftrightarrow$ (v) hold also for separable Orlicz spaces on the interval $[0,1)$ equipped with the Luxemburg norm. A proof of this result, also based on Theorem 2.7, is given in [MS], Theorem 3.

\section{StRictly MONOTONE SYMmetRIC NORMS}

In this section, we present a characterization of the Kadec-Klee property for convergence in measure via a monotonicity condition which relates the natural order structure of a symmetric space with its metric structure. The relevant definitions follow. Let $\left(E,\|\cdot\|_{E}\right)$ be a symmetric space on $([0, \alpha), m)$. The norm $\|\cdot\|_{E}$ on $E$ is said to be strictly monotone if and only if

$$
x, y \in E, 0 \leq x \leq y, x \neq y \Rightarrow\|x\|_{E}<\|y\|_{E} .
$$

The norm $\|\cdot\|_{E}$ on $E$ is said to be locally uniformly strictly monotone if and only if

$$
x \in E,\left\{x_{n}\right\} \subseteq E, 0 \leq x \leq x_{n}, n=1,2, \ldots,\left\|x_{n}\right\|_{E} \rightarrow\|x\|_{E} \Rightarrow\left\|x_{n}-x\right\|_{E} \rightarrow 0 .
$$

It is not without interest to observe that local uniform strict monotonicity of the norm on $E$ is in fact equivalent to the following property, which is a local version of a uniform monotonicity condition introduced by Birkhoff [Bi] : for all $\epsilon>0$ and $0 \leq x \in E$, there exists $\delta_{E}(x, \epsilon)>0$ such that for all $0 \leq y \in E$, whenever $\|x+y\|_{E} \leq 1+\delta_{E}(x, \epsilon)$, it follows that $\|y\|_{E}<\epsilon$.

Let us observe that if $x, y \in E, 0 \leq x \leq y, x \neq y$ and $y^{*}(\infty)=0$, then $0 \leq x^{*} \leq y^{*}$ and $x^{*} \neq y^{*}$. It follows that if $E$ is separable, then $E$ has strictly monotone norm if and only if

$$
x, y \in E, 0 \leq x^{*} \leq y^{*}, x^{*} \neq y^{*} \Rightarrow\|x\|_{E}<\|y\|_{E} .
$$

We shall use this remark without further reference. The uniform version is somewhat less trivial and is the equivalence of statements (ii), (iii) of Theorem 3.2 below. We shall need some further notation. If $y \in \Lambda_{1}[0, \infty)$ and if $\tau>0$, we set

$$
y_{\tau}(s)= \begin{cases}0, & \text { if } s<\tau ; \\ y(s-\tau), & \text { if } s \geq \tau\end{cases}
$$

To smoothen the presentation of the main result of this section, we require the following preliminary result.

Lemma 3.1. Let $0 \leq x=x^{*}, z \in \Lambda_{1}[0, \infty)$. If $\tau>0$, and if $z \chi_{[\tau, \infty)}=0$, then

$$
x+z_{\tau} \prec \prec x+z .
$$

Proof. Let $t>0$ and let $T \subseteq[0, \infty)$ be measurable with $m(T)=t$. We set

$$
T_{1}=T \cap[0, \tau), T_{2}=T \cap[\tau, 2 \tau), T_{3}=T \cap[2 \tau, \infty) .
$$


Let $Q=T_{1} \cup\left(T_{2}-\tau\right) \cup\left(T_{2} \cap\left(T_{1}+\tau\right)\right)$ and observe that $m(Q)=m\left(T_{1} \cup T_{2}\right)$. It follows that

$$
\begin{aligned}
\int_{Q}(x+z)(s) d s & =\int_{T_{1} \backslash\left(T_{2}-\tau\right)}(x+z)(s) d s+\int_{T_{1} \cap\left(T_{2}-\tau\right)}(x+z)(s) d s \\
& +\int_{\left(T_{2}-\tau\right) \backslash T_{1}}(x+z)(s) d s+\int_{T_{2} \cap\left(T_{1}+\tau\right)} x(s) d s \\
& \geq \int_{T_{1} \backslash\left(T_{2}-\tau\right)} x(s) d s+\int_{T_{1} \cap\left(T_{2}-\tau\right)} x(s) d s+\int_{T_{1} \cap\left(T_{2}-\tau\right)} z(s) d s \\
& +\int_{\left(T_{2}-\tau\right) \backslash T_{1}} x(s) d s+\int_{\left(T_{2}-\tau\right) \backslash T_{1}} z(s) d s+\int_{T_{2} \cap\left(T_{1}+\tau\right)} x(s) d s \\
& =\int_{T_{1}} x(s) d s+\int_{\left(T_{1}+\tau\right) \cap T_{2}} z_{\tau}(s) d s+\int_{\left(T_{2}-\tau\right) \backslash T_{1}} x(s) d s \\
& +\int_{T_{2} \backslash\left(T_{1}+\tau\right)} z_{\tau}(s) d s+\int_{T_{2} \cap\left(T_{1}+\tau\right)} x(s) d s \\
& =\int_{T_{1}} x(s) d s+\int_{\left(T_{1}+\tau\right) \cap T_{2}}\left(x+z_{\tau}\right)(s) d s \\
& +\int_{\left(T_{2}-\tau\right) \backslash T_{1}} x(s) d s+\int_{T_{2} \backslash\left(T_{1}+\tau\right)} z_{\tau}(s) d s \\
& \geq \int_{T_{1}} x(s) d s+\int_{\left(T_{1}+\tau\right) \cap T_{2}}\left(x+z_{\tau}\right)(s) d s \\
& +\int_{T_{2} \backslash\left(T_{1}+\tau\right)} z_{\tau}(s) d s+\int_{T_{2} \backslash\left(T_{1}+\tau\right)} x(s) d s \\
& =\int_{T_{1} \cup T_{2}}\left(x+z_{\tau}\right)(s) d s . \\
& o b t a i n \\
& \\
& \\
&
\end{aligned}
$$

Since $z_{\tau} \chi_{[2 \tau, \infty)}=0$, we obtain

$$
\int_{Q \cup T_{3}}(x+z)(s) d s \geq \int_{T_{1} \cup T_{2} \cup T_{3}}\left(x+z_{\tau}\right)(s) d s=\int_{T}\left(x+z_{\tau}\right)(s) d s .
$$

Since $m\left(Q \cup T_{3}\right)=t$ it follows that

$$
\int_{T}\left(x+z_{\tau}\right)(s) d s \leq \int_{[0, t)}(x+z)^{*}(s) d s
$$

and this suffices to complete the proof since

$$
\int_{[0, t)}\left(x+z_{\tau}\right)^{*}(s) d s=\sup \left\{\int_{T}\left(x+z_{\tau}\right)(s) d s: m(T)=t\right\} .
$$

The principal result of this section now follows.

Theorem 3.2. (a) If $\left(E,\|\cdot\|_{E}\right)$ is a separable symmetric space on $([0, \alpha), m)$, then the following statements are equivalent.

(i) The norm $\|\cdot\|_{E}$ on $E$ is strictly monotone and $\left(E,\|\cdot\|_{E}\right)$ has the Kadec-Klee property for convergence in measure.

(ii) The norm $\|\cdot\|_{E}$ on $E$ is locally uniformly strictly monotone. 
(iii) If $x \in E,\left\{x_{n}\right\} \subseteq E$ satisfy $0 \leq x^{*} \leq x_{n}^{*}, n=1,2, \ldots$ and if $\left\|x_{n}\right\|_{E} \rightarrow\|x\|_{E}$, then $\left\|x_{n}^{*}-x^{*}\right\|_{E} \rightarrow 0$.

(b) If $\left(E,\|\cdot\|_{E}\right)$ is a separable symmetric space on $([0, \infty), m)$, then each of the preceding statements is equivalent to

(iv) E has the Kadec-Klee property for local convergence in measure.

Proof. Throughout we will assume $\alpha=\infty$, since the argument in this case contains that for the case that $\alpha<\infty$.

(i) $\Rightarrow$ (iii) Suppose that $x, x_{n} \in E$, that $x^{*} \leq x_{n}^{*}, n=1,2, \ldots$ and that $\left\|x_{n}\right\|_{E} \rightarrow\|x\|_{E}$. As in the proof of Proposition 2.4, we may apply the Helly selection principle, and by passing to a subsequence and relabelling if necessary, we may assume that the sequence $\left\{x_{n}^{*}\right\}$ converges pointwise to some non-increasing function $z$ with $z \geq x^{*}$. Lower-semicontinuity of the norm for pointwise convergence implies that $\|z\|_{E}=\|x\|_{E}$ and strict monotonicity of the norm then implies that $z=x^{*}$. Since $x^{*}(\infty)=0$, it follows that the sequence $\left\{x_{n}^{*}\right\}$ converges to $x^{*}$ in measure, and the assumption that $E$ has the Kadec-Klee property for convergence in measure now implies that $\left\|x_{n}^{*}-x^{*}\right\|_{E} \rightarrow 0$.

(iii) $\Rightarrow$ (i). It follows immediately from the assertion of (iii) that if $x, y \in E, x^{*} \leq$ $y^{*}$ and $\|x\|_{E}=\|y\|_{E}$, then $y^{*}=x^{*}$ and so the norm on $E$ is strictly monotone. Now suppose that $x \in E,\left\{x_{n}\right\} \subseteq E$, that $\left\{x_{n}\right\}$ converges to $x$ in measure and that $\left\|x_{n}\right\|_{E} \rightarrow\|x\|_{E}$. Since $\left\{x_{n}^{*}\right\}$ converges to $x^{*} m$-almost everywhere (see [KPS], Chapter II, 2.11), it follows that

$$
\sup _{k \geq n}\left(x^{*}-x_{k}^{*}\right)^{+} \downarrow_{n} 0 .
$$

Consequently, by order-continuity of the norm on $E$, it follows that

$$
\left\|\left(x^{*}-x_{n}^{*}\right)^{+}\right\|_{E} \leq\left\|\sup _{k \geq n}\left(x^{*}-x_{k}^{*}\right)^{+}\right\|_{E} \downarrow_{n} 0 .
$$

The inequalities

$$
x_{n}^{*} \leq \max \left\{x_{n}^{*}, x^{*}\right\} \leq x_{n}^{*}+\sup _{k \geq n}\left(x^{*}-x_{k}^{*}\right)^{+}, \quad n=1,2, \ldots,
$$

together with (3.1) imply that $\left\|\max \left\{x_{n}^{*}, x^{*}\right\}\right\|_{E} \rightarrow\|x\|_{E}$ and so (iii) implies that $\left\|\max \left\{x_{n}^{*}, x^{*}\right\}-x^{*}\right\|_{E} \rightarrow 0$. Since

$$
\left\|\max \left\{x_{n}^{*}, x^{*}\right\}-x^{*}\right\|_{E}=\left\|\left(x_{n}^{*}-x^{*}\right)^{+}\right\|_{E},
$$

it follows that $\left\|\left(x_{n}^{*}-x^{*}\right)^{+}\right\|_{E} \rightarrow 0$ and combining this with (3.1), it follows also that $\left\|x_{n}^{*}-x^{*}\right\|_{E} \rightarrow 0$. That $E$ has the Kadec-Klee property for convergence in measure now follows from the implication (iii) $\Rightarrow$ (i) of Corollary 1.6.

The implication (ii) $\Rightarrow$ (iii) is trivial.

(iii) $\Rightarrow$ (ii). We suppose then that $x, x_{n} \in E$, that $0 \leq x \leq x_{n}, n=1,2, \ldots$ and that $\left\|x_{n}\right\|_{E} \rightarrow\|x\|_{E}$. It follows that $0 \leq x^{*} \leq x_{n}^{*}, n=1,2, \ldots$ and that $\left\|x_{n}^{*}\right\|_{E} \rightarrow\left\|x^{*}\right\|_{E}$, and (ii) implies that $\left\|x_{n}^{*}-x^{*}\right\|_{E} \rightarrow 0$. Via Corollary 1.6(iii), it suffices to show that $\left\{x_{n}\right\}$ converges to $x$ in measure. If this is not the case, then passing to a subsequence and relabelling if necessary, we may assume that there exist positive numbers $\epsilon, \delta$ with $0<\delta<\epsilon<1$ such that

$$
m\left(\left\{x_{n}>x+2 \epsilon\right\}\right)>2 \delta, \quad n=1,2, \ldots
$$


Since $x^{*}(\infty)=0$, there exists $\gamma>1$ such that

$$
x^{*}\left(\frac{\gamma-\delta}{2}\right)<\frac{\epsilon}{2}
$$

Let $0 \leq y \in E$ be a (countably-valued ) simple function such that $0 \leq z=y-x \leq$ $(2 \gamma)^{-1} \epsilon \delta$. There exists a measure preserving isomorphism $\pi$ from $[0, \alpha)$ onto $[0, \alpha)$ such that $y^{*}=y \circ \pi$. We set $T w=w \circ \pi, w \in E$ and

$$
e_{n}=\left\{t \in[0, \alpha): T\left(x_{n}+z\right)(t)>T(x+z)(t)+2 \epsilon\right\}, \quad n=1,2, \ldots
$$

It is clear that $m\left(e_{n}\right)>2 \delta$ and so for each $n=1,2, \ldots$, either $m\left(e_{n} \cap[0, \gamma)\right)>\delta$ or $m\left(e_{n} \cap[\gamma, \infty)\right)>\delta, n=1,2, \ldots$ If $m\left(e_{n} \cap[0, \gamma)\right)>\delta$, then

$$
\begin{aligned}
\int_{0}^{\gamma}\left(x_{n}+z\right)^{*}(t) d t & =\int_{0}^{\gamma}\left(T\left(x_{n}+z\right)\right)^{*}(t) d t \\
& \geq \int_{0}^{\gamma} T\left(x_{n}+z\right)(t) d t \\
& =\int_{e_{n} \cap[0, \gamma)}^{\gamma} T\left(x_{n}+z\right)(t) d t+\int_{[0, \gamma) \backslash e_{n}} T\left(x_{n}+z\right)(t) d t \\
& \geq \int_{0}^{\gamma} T(x+z)(t) d t+2 \epsilon \delta \\
& =\int_{0}^{\gamma} y^{*}(t) d t+2 \epsilon \delta
\end{aligned}
$$

On the other hand, if $m\left(e_{n} \cap[\gamma, \infty)\right)>\delta$, we choose $f_{n} \subseteq e_{n} \cap[\gamma, \infty)$ such that $m\left(f_{n}\right)=\delta$. Using Lemma 16 of $[\mathrm{Fr}]$, we obtain that

$$
\begin{aligned}
\int_{0}^{\gamma}\left(x_{n}+z\right)^{*}(t) d t & =\int_{0}^{\gamma}\left(T\left(x_{n}+z\right)\right)^{*}(t) d t \\
& \geq \int_{0}^{\gamma-\delta}\left(T\left(x_{n}+z\right) \chi_{f_{n}^{c}}\right)^{*}(t) d t+\int_{0}^{\delta}\left(T\left(x_{n}+z\right) \chi_{f_{n}}\right)^{*}(t) d t \\
& \geq \int_{0}^{\gamma-\delta} T\left(x_{n}+z\right)(t) d t+\int_{f_{n}} T\left(x_{n}+z\right)(t) d t \\
& \geq \int_{0}^{\gamma-\delta} y^{*}(t) \chi_{f_{n}^{c}} d t+\int_{f_{n}}\left(y^{*}+2 \epsilon\right)(t) d t \\
& \geq \int_{0}^{\gamma-\delta} y^{*}(t) d t+2 \epsilon \delta
\end{aligned}
$$

Since

$$
y^{*}(\gamma-\delta) \leq x^{*}\left(2^{-1}(\gamma-\delta)\right)+z^{*}\left(2^{-1}(\gamma-\delta)\right)<\epsilon
$$

it follows that

$$
2 \epsilon \delta \geq y^{*}(\gamma-\delta) \delta+\epsilon \delta \geq \int_{\gamma-\delta}^{\gamma} y^{*}(t) d t+\epsilon \delta
$$

so that

$$
\int_{0}^{\gamma}\left(x_{n}+z\right)^{*}(t) d t \geq \int_{0}^{\gamma} y^{*}(t) d t+\epsilon \delta
$$


Consequently, it follows that

$$
\begin{aligned}
\int_{0}^{\gamma} x^{*}(t) d t+\epsilon \delta & \leq \int_{0}^{\gamma} y^{*}(t) d t+\epsilon \delta \\
& \leq \int_{0}^{\gamma}\left(x_{n}+z\right)^{*}(t) d t \\
& \leq \int_{0}^{\gamma} x_{n}^{*}(t) d t+\int_{0}^{\gamma} z^{*}(t) d t \\
& \leq \int_{0}^{\gamma} x_{n}^{*}(t) d t+2^{-1} \epsilon \delta, \quad n=1,2, \ldots,
\end{aligned}
$$

and this contradicts the fact that $\left\|x_{n}^{*}-x^{*}\right\|_{E} \rightarrow 0$. This proves the implication (iii) $\Rightarrow$ (ii), and suffices to prove the assertion of (a).

(iv) $\Rightarrow$ (i). We assume that $E$ has the Kadec-Klee property for local convergence in measure. It clearly suffices to show that the norm on $E$ is strictly monotone. We suppose that $x=x^{*}, y=y^{*} \in E$, that $x^{*} \leq y^{*}, x^{*} \neq y^{*}$ and that $\|x\|_{E}=\|y\|_{E}$. We suppose that $t_{0}>0$ is such that $x^{*}\left(t_{0}\right)<y^{*}\left(t_{0}\right)$. We let $N:=\left[t_{0}+1\right]$ and set

$$
y_{1}=y \chi_{[0, N)}+x \chi_{[N, \infty)} .
$$

It is clear that $y_{1}^{*}=y_{1}$, that $x \leq y_{1} \leq y$, that $x \neq y_{1}$ and that

$$
\|x\|_{E}=\left\|y_{1}\right\|_{E}=\|y\|_{E} .
$$

If we set $z:=y_{1}-x$, then it is clear that $z$ is supported by the interval $[0, N)$. By Lemma 3.1, it follows that

$$
x+z_{n} \prec \prec x+z=y_{1}
$$

for all $n>N$ where

$$
z_{n}(s)= \begin{cases}0, & \text { if } s<n \\ z(s-n), & \text { if } s \geq n,\end{cases}
$$

for all $n=1,2, \ldots$. It is clear that the sequence $\left\{x+z_{n}\right\}$ converges to $x$ locally in measure. By separability of $E$ (see Proposition 1.1), it follows that

$$
\|x\|_{E} \leq\left\|x+z_{n}\right\|_{E} \leq\left\|y_{1}\right\|_{E}=\|x\|_{E}, \quad n>N .
$$

Since $\left(E,\|\cdot\|_{E}\right)$ has the Kadec-Klee property for local convergence in measure, it follows that $\left\|z_{n}\right\|_{E} \rightarrow 0$. Since $\|z\|_{E}=\left\|z_{n}\right\|_{E}$, it follows also that $z=0$. This implies that $x=y$, contrary to assumption, and completes the proof of the implication (iv) $\Rightarrow$ (i).

To complete the proof of the theorem, it will suffice to prove the implication (ii) $\Rightarrow$ (iv). We assume then that the norm on $E$ is locally uniformly strictly monotone. Let $x \in E,\|x\|_{E}=1$, and suppose that $\left\{x_{n}\right\} \subseteq E$ satisfies $\left\|x_{n}\right\|_{E} \rightarrow 1$ and $x_{n} \rightarrow x(\mathrm{~lm})$. By Lemma 1.8 and passing to a subsequence if necessary, there exist measurable subsets $e_{n} \subseteq[0, \infty), n \geq 1$, of finite measure such that $\chi_{e_{n}} \longrightarrow 1$ locally in measure and

$$
\left\|x_{n} \chi_{e_{n}}-x\right\|_{E} \rightarrow 0, \quad\left\|x \chi_{f_{n}}\right\|_{E} \rightarrow 0
$$

where $f_{n}=[0, \infty) \backslash e_{n}, n \geq 1$. If we set $y_{n}=x_{n} \chi_{f_{n}}$ for all $n \geq 1$, then it suffices to show that $\left\|y_{n}\right\|_{E} \rightarrow 0$. If this is not so, then it follows that $\left\{y_{n}\right\}$ does not converge to zero in measure, since $E$ has the Kadec-Klee property for convergence in measure by the implication (ii) $\Rightarrow(\mathrm{i})$ and $x_{n} \chi_{e_{n}} \rightarrow x$ in measure. Consequently, 
there exists a positive number $\epsilon>0$ and measurable subsets $f_{n}^{\prime} \subseteq f_{n}, n \geq 1$, such that $m\left(f_{n}^{\prime}\right)=\epsilon$ and $\left|y_{n}\right| \chi_{f_{n}^{\prime}} \geq \epsilon \chi_{f_{n}^{\prime}}, n \geq 1$. We set

$$
v_{n}=\left|x_{n}\right| \chi_{e_{n}}+\left|x-x_{n}\right| \chi_{e_{n}}+|x| \chi_{f_{n}}+\epsilon \chi_{f_{n}^{\prime}}, \quad n \geq 1
$$

It is clear that $0 \leq|x| \leq v_{n}, \quad n \geq 1$. From the inequalities

$$
\left|x_{n}\right| \chi_{e_{n}} \leq\left|x_{n}\right| \chi_{e_{n}}+\epsilon \chi_{f_{n}^{\prime}} \leq\left|x_{n}\right|, \quad n \geq 1
$$

it follows also that $\left\|v_{n}\right\|_{E} \rightarrow 1$. By local uniform strict monotonicity of the norm on $E$, it now follows that $\left\|v_{n}-|x|\right\|_{E} \rightarrow 0$, but by arguing similarly to the proof of 2.7 (ii) $\Rightarrow$ (iii), it can be seen that this is not the case. This suffices to complete the proof of the theorem.

The authors wish to thank the referee for pointing out a gap in the proof of the implication (iv) $\Rightarrow$ (i) in the original version of this paper.

Let us observe that the two properties of assertion (i) of Theorem 3.2 are independent. In fact, if $E$ is separable, then it is clear that if the norm on $E$ is strictly $K$-monotone, then the norm on $E$ is strictly monotone. Accordingly, Example 2.8 now yields an example of a separable symmetric space with strictly monotone norm but without the Kadec-Klee property for convergence in measure. On the other hand, if $\phi \in \Phi$ and if $\phi^{\prime}=\phi^{\prime} \chi_{[0, \gamma)}$ for some $0<\gamma<\alpha$, then the Lorentz space $\left(\Lambda_{\phi},\|\cdot\|_{\Lambda_{\phi}}\right)$ on $[0, \alpha)$ has the Kadec-Klee property for convergence in measure, by Corollary 1.3, but does not have strictly monotone norm. By contrast, any symmetric space on $([0, \infty), m)$ with the Kadec-Klee property for local convergence in measure does have strictly monotone norm (see $[\mathrm{Ar}]$ for the analogous result in the setting of symmetric sequence spaces).

Theorem 3.3. If $\Lambda_{\phi}$ is any Lorentz space on $[0, \infty)$, then $\Lambda_{\phi}$ has the Kadec-Klee property for local convergence in measure if and only if $\Lambda_{\phi}$ is separable.

Proof. By Proposition 1.1, it suffices to show that if $\Lambda_{\phi}$ is separable, then $\Lambda_{\phi}$ has the Kadec-Klee property for local convergence in measure. Assume then that $\Lambda_{\phi}$ is separable, so that $\phi(\infty)=\infty$ and let $x \in \Lambda_{\phi},\left\{x_{n}\right\} \subseteq \Lambda_{\phi}$, satisfy $0 \leq x^{*} \leq x_{n}^{*}, n \geq 1$ and $\left\|x_{n}\right\|_{\Lambda_{\phi}} \rightarrow\|x\|_{\Lambda_{\phi}}$. By Theorem 3.2, it suffices to show that $\left\|x_{n}^{*}-\bar{x}^{*}\right\|_{\Lambda_{\phi}} \rightarrow 0$. By Corollary 1.3, we have to check only that $x_{n}^{*}-x^{*} \rightarrow 0$ in measure. Set $y_{n}=$ $x_{n}^{*}-x^{*}, n \geq 1$. If $y_{n} \nrightarrow 0$ in measure, then we may assume that there exists $\epsilon>0$ such that $m\left(\left\{y_{n}>\epsilon\right\}\right)>\epsilon$ for all $n \geq 1$. Set $e_{n}=\left\{y_{n}>\epsilon\right\}, n \geq 1$. Either (i) $m\left(e_{n} \cap[0, t)\right) \rightarrow 0$ for every $0<t$ for which $x^{*}(t)<\epsilon / 4$ or (ii) there exists $0<t_{0}$ with $x^{*}\left(t_{0}\right)<\epsilon / 4$ and $\delta>0$ such that $m\left(e_{n} \cap\left[0, t_{0}\right)\right) \geq \delta$ for infinitely many natural numbers $n$. We suppose first that (ii) holds and without loss of generality, we suppose that there exists $t_{0}>0$ with $x^{*}\left(t_{0}\right)<\epsilon / 4$ and $\delta>0$ such that $m\left(e_{n} \cap\left[0, t_{0}\right)\right) \geq \delta$ for all $n \geq 1$. We observe that

$$
\begin{aligned}
\left\|x_{n}\right\|_{\Lambda_{\phi}} & \geq \int_{[0, \infty)} x^{*}(t) \phi^{\prime}(t) d t+\int_{[0, \infty)} y_{n}(t) \phi^{\prime}(t) d t \\
& \geq\|x\|_{\Lambda_{\phi}}+\int_{e_{n} \cap\left[0, t_{0}\right)} y_{n}(t) \phi^{\prime}(t) d t \\
& \geq\|x\|_{\Lambda_{\phi}}+\epsilon\left(\phi\left(t_{0}\right)-\phi\left(t_{0}-\delta\right)\right),
\end{aligned}
$$


for all $n \geq 1$. This however contradicts the assumption that $\left\|x_{n}\right\|_{\Lambda_{\phi}} \rightarrow\|x\|_{\Lambda_{\phi}}$ since the concavity of $\phi$ and the assumption that $\phi(\infty)=\infty$ imply that $\phi\left(t_{0}\right)-\phi\left(t_{0}-\delta\right)>$ 0 .

We now may assume that (i) holds and let $0<t_{0}$ satisfy $x^{*}\left(t_{0}\right)<\epsilon / 4$. Without loss of generality, we may assume that

$$
m\left(e_{n} \cap\left[0, t_{0}\right)\right)<\epsilon / 4, \quad m\left(e_{n} \cap\left[0, t_{0}+1\right)\right)<\epsilon / 4
$$

for all $n \geq 1$. It then follows that

$$
x_{n}^{*}\left(t_{0}\right)-x^{*}\left(t_{0}\right)>3 \epsilon / 4, \quad n \geq 1 .
$$

In fact, if $x_{n}^{*}\left(t_{0}\right)<x^{*}\left(t_{0}\right)+3 \epsilon / 4$, then $x_{n}^{*}(t)<\epsilon$ for all $t \geq t_{0}$ so that

$$
m\left(e_{n}\right)=m\left(e_{n} \cap\left[0, t_{0}\right)\right)+m\left(e_{n} \cap\left[t_{0}, \infty\right)\right)<\epsilon / 4 .
$$

Similarly it follows that

$$
x_{n}^{*}\left(t_{0}+1\right)-x^{*}\left(t_{0}+1\right)>3 \epsilon / 4, \quad n \geq 1 .
$$

Consequently, if $t \in\left[t_{0}, t_{0}+1\right)$, then

$$
x_{n}^{*}(t)-x^{*}(t) \geq x_{n}^{*}\left(t_{0}+1\right)-x^{*}\left(t_{0}\right) \geq \epsilon / 2 .
$$

As in the first part of the proof we obtain that

$$
\left\|x_{n}\right\|_{\Lambda_{\phi}} \geq\|x\|_{\Lambda_{\phi}}+\frac{\epsilon}{2}\left(\phi\left(t_{0}+1\right)-\phi\left(t_{0}\right)\right)
$$

and this again contradicts the assumption that $\left\|x_{n}\right\|_{\Lambda_{\phi}} \rightarrow\|x\|_{\Lambda_{\phi}}$.

\section{Rotundity}

We recall that a Banach space $X$ is called rotund if and only if

$$
x, y \in X, \quad x \neq y, \quad\|x\|_{X}=\|y\|_{X}=1 \Rightarrow\|x+y\|_{X}<2 ;
$$

$X$ is called mid-point locally uniformly rotund if and only if $x \in X, \quad\left\{x_{n}\right\},\left\{y_{n}\right\} \subseteq$ $X$,

$$
\|x\|_{X}=1,\left\|x_{n}\right\|_{X},\left\|y_{n}\right\|_{X} \rightarrow 1,\left\|2 x-\left(x_{n}+y_{n}\right)\right\|_{X} \rightarrow 0 \Rightarrow\left\|x_{n}-y_{n}\right\|_{X} \rightarrow 0 ;
$$

$X$ is called weakly mid-point locally uniformly rotund if and only if $x \in X,\left\{x_{n}\right\}$, $\left\{y_{n}\right\} \subseteq X$

$$
\|x\|_{X}=1,\left\|x_{n}\right\|_{X} \rightarrow 1,\left\|y_{n}\right\|_{X} \rightarrow 1, x_{n}+y_{n} \rightarrow 2 x \sigma\left(X, X^{*}\right) \Rightarrow\left\|x_{n}-y_{n}\right\|_{X} \rightarrow 0 .
$$

The notion of mid-point local uniform rotundity was introduced by Anderson [An] while that of weak mid-point local uniform rotundity appears in Godefroy [Go] . It has been shown by Anderson [An] that the Kadec-Klee property implies midpoint local uniform rotundity for rotund reflexive spaces, while the same result has been shown to hold by Kadec [Ka2] for rotund spaces which contain no isomorphic copy of $l_{1}$. By contrast, we show here that mid-point local uniform rotundity is equivalent to the Kadec-Klee property with respect to $\Lambda_{\infty}$ (respectively, the KadecKlee property for local convergence in measure) for separable rotund symmetric spaces on $([0, \alpha), m)$ which are dual spaces. This is an immediate consequence of Theorem 4.1 following. As Example 4.2 below shows, such spaces may contain isomorphic copies of $l_{1}$. The theorem which follows is an extension and refinement of [Su], Theorem 1. 
Theorem 4.1. Let $\left(E,\|\cdot\|_{E}\right)$ be a separable symmetric space on $([0, \alpha), m)$ and consider the following statements.

(i) $E$ has the Kadec-Klee property with respect to $\Lambda_{\infty}$.

(ii) E has the Kadec-Klee property for convergence in measure.

(iii) E has the Kadec-Klee property for local convergence in measure.

(iv) The norm on $E$ is locally uniformly strictly monotone.

(v) The norm on $E$ is locally uniformly strictly $K$-monotone.

(vi) E is mid-point locally uniformly rotund.

(vii) $E$ is weakly mid-point locally uniformly rotund.

If $E$ is rotund then statements (i)-(v) are equivalent and each of these statements is implied by (vi). If in addition $E$ is the dual space of some separable symmetric space $F$ on $([0, \alpha), m)$, then all statements (i)-(vii) are equivalent.

Proof. It is easy to see that rotundity of the norm on $E$ implies that the norm on $E$ is strictly $K$-monotone and therefore strictly monotone. The equivalence of (i)-(v) then is an immediate consequence of Theorems 2.7, 3.2. The remaining assertions are proved exactly as in $[\mathrm{Su}]$, Theorem 1 . For the sake of completeness, we indicate the details. We show first the implication (vi) $\Rightarrow\left(\right.$ iv). Let $0 \leq x \leq x_{n} \in E, n=$ $1,2, \ldots$ and suppose that $\left\|x_{n}\right\|_{E} \rightarrow\|x\|_{E}=1$. If we set $y_{n}=2 x-x_{n}, n=1,2, \ldots$, then $\left|y_{n}\right| \leq x_{n}, n=1,2, \ldots$ and the inequalities

$$
2-\left\|x_{n}\right\|_{E} \leq\left\|y_{n}\right\|_{E} \leq\left\|x_{n}\right\|_{E}, \quad n=1,2, \ldots,
$$

imply that $\left\|y_{n}\right\|_{E} \rightarrow 1$. Since

$$
x_{n}+y_{n}=2 x, \quad n=1,2, \ldots,
$$

it follows from the assumed mid-point local uniform rotundity of $E$ that $\left\|x_{n}-x\right\|_{E}=$ $2^{-1}\left\|x_{n}-y_{n}\right\|_{E} \rightarrow 0$. We assume finally that $E$ is the dual space of some separable symmetric space $F$ on $([0, \alpha), m)$, and prove the implication (i) $\Rightarrow$ (vii). We suppose that $x \in E$, that $\left\{x_{n}\right\},\left\{y_{n}\right\}$ are sequences in $E$ with $\|x\|_{E}=1,\left\|x_{n}\right\|_{E} \rightarrow$ $1,\left\|y_{n}\right\|_{E} \rightarrow 1$ and that the sequence $\left\{x_{n}+y_{n}\right\}$ converges weakly to $2 x$. By separability of $F$, the unit ball of $E$ is compact and metrizable for the $\sigma(E, F)$ topology. Consequently, there exist $u, v \in E$ and subsequences $\left\{x_{n(k)}\right\} \subseteq\left\{x_{n}\right\},\left\{y_{n(k)}\right\} \subseteq\left\{y_{n}\right\}$ such that the sequences $\left\{x_{n(k)}\right\},\left\{y_{n(k)}\right\}$ are $\sigma(E, F)$-convergent to $u, v$ respectively. It follows that $2 x=u+v$ and so the assumption that the norm on $E$ is rotund implies that $u=v=x$. Since $E$ has the Kadec-Klee property with respect to $\Lambda_{\infty}$, it follows that $\left\|x_{n(k)}-x\right\|_{E} \rightarrow 0$ and $\left\|y_{n(k)}-x\right\|_{E} \rightarrow 0$ so that $\left\|x_{n(k)}-y_{n(k)}\right\|_{E} \rightarrow 0$. This suffices to complete the proof of the theorem.

The Banach space $X$ is called locally uniformly convex if and only if whenever $x \in$ $X$ and $\left\{x_{n}\right\}$ is a sequence in $X$ such that $\|x\|_{X}=1,\left\|x_{n}\right\|_{X} \rightarrow 1$ and $\left\|x+x_{n}\right\|_{X} \rightarrow 2$, it follows that $\left\|x-x_{n}\right\|_{X} \rightarrow 0$. It is well known that local uniform convexity implies mid-point local uniform rotundity [Sm]. The example which follows (see also [Su] ) shows that the converse implication may fail for symmetric spaces which are rotund dual spaces. In particular, statement (vi) in the preceding Theorem 4.1 cannot be replaced by the assertion that $E$ is locally uniformly convex, as is the case with Orlicz spaces equipped with the Luxemburg norm [MS] .

Example 4.2. If $\phi(t)=t^{\frac{1}{4}}, t>0$, we let $E$ be $\Lambda_{\phi}$ on $[0,1)$, equipped with the norm

$$
\|\cdot\|_{E}=\|\cdot\|_{\Lambda_{\phi}}+\|\cdot\|_{2} .
$$


It follows from $[\mathrm{BS}]$, Theorem 5.13 that $\left(\Lambda_{\phi},\|\cdot\|_{\Lambda_{\phi}}\right)$ embeds continuously with norm one into $\left(L_{2}[0,1),\|\cdot\|_{2}\right)$, and from this it follows that $\|\cdot\|_{E}$ is equivalent to $\|\cdot\|_{\Lambda_{\phi}}$. It is not difficult to check that $\left(E,\|\cdot\|_{E}\right)$ is rotund. To show that the norm on $E$ is locally uniformly strictly $K$-monotone, note that if $x \prec \prec x_{n} \in$ $E, n=1,2, \ldots$, and $\left\|x_{n}\right\|_{E} \rightarrow\|x\|_{E}$, then also $\left\|x_{n}\right\|_{\Lambda_{\phi}} \rightarrow\|x\|_{\Lambda_{\phi}}$. From the strict concavity of $\phi$ and Theorem 2.11, it follows that $\left\|x_{n}-x\right\|_{\Lambda_{\phi}} \stackrel{\rightarrow}{\rightarrow} 0$, and therefore also $\left\|x_{n}-x\right\|_{E} \rightarrow 0$. That $E$ is a dual space follows from [KPS], Theorem 5.4 of Chapter II. To show that $\left(E,\|\cdot\|_{E}\right)$ is not locally uniformly convex, set $x=\frac{1}{2} \chi_{[0,1)}$ and $x_{n}=n^{1 / 4} \chi_{[0,1 / n)}, n=1,2, \ldots$ It is straightforward to verify that $\|x\|_{E}=1$, that $\left\|x_{n}\right\|_{E} \rightarrow 1$ and $\left\|x+x_{n}\right\|_{E} \rightarrow 2$ but that $\left\|x-x_{n}\right\|_{E} \nrightarrow 0$.

Finally, we give an example of a separable symmetric space which is rotund but which does not have the Kadec-Klee property.

Example 4.3. Let $\psi(\tau)=\frac{3}{2} \tau^{2 / 3}, \tau>0$ and let $\left(M_{\psi},\|\cdot\|_{M_{\psi}}\right)$ be the corresponding Marcinkiewicz space on $([0,1), m)$ consisting of all $x \in \Lambda_{1}$ for which

$$
\|x\|_{M_{\psi}}=\sup _{0<\tau<1}[\psi(\tau)]^{-1} \int_{[0, \tau)} x^{*}(s) d s<\infty .
$$

Since the unit sphere of $M_{\psi}$ consists of all $x \in \Lambda_{1}$ such that $x \prec \prec \psi^{\prime}$, it follows immediately that $\left(M_{\psi},\|\cdot\|_{M_{\psi}}\right)$ embeds continuously with norm one into $\left(L_{2}[0,1)\right.$, $\left.\|\cdot\|_{2}\right)$. We define $\left(E,\|\cdot\|_{E}\right)$ to be $M_{\psi}^{0}$ equipped with the norm

$$
\|\cdot\|_{E}=\|\cdot\|_{M_{\psi}}+\|\cdot\|_{2},
$$

where $M_{\psi}^{0}$ is the set of all $x \in M_{\psi}$ for which

$$
\lim _{\tau \rightarrow 0}[\psi(\tau)]^{-1} \int_{[0, \tau)} x^{*}(s) d s=0 .
$$

The space $\left(E,\|\cdot\|_{E}\right)$ is separable and it is not difficult to see that $\left(E,\|\cdot\|_{E}\right)$ is rotund. If

$$
x=\chi_{[0,1)}, \quad y_{n}=n^{1 / 3} \chi_{[0,1 / n)}, \quad x_{n}=x+y_{n}, \quad n=1,2, \ldots,
$$

then a straightforward computation shows that $\left\|x_{n}\right\|_{E} \rightarrow\|x\|_{E}$. Since $\left\|y_{n}\right\|_{1} \rightarrow 0$ it follows that $x_{n} \rightarrow x \sigma\left(E, \Lambda_{\infty}\right)$ and since $E^{*}$ is isomorphic to the separable Lorentz space $\Lambda_{\psi}$ (see [KPS], Theorem 5.4 of Chapter II), it follows also that $\left\{x_{n}\right\}$ converges to $x$ weakly. However,

$$
\left\|x_{n}-x\right\|_{E} \geq \sup _{0<\tau<1} \frac{2}{3} \tau^{-2 / 3} \int_{[0, \tau)} n^{1 / 3} \chi_{[0,1 / n)}(s) d s \geq \frac{2}{3}, \quad n=1,2, \ldots,
$$

and so $\left(E,\|\cdot\|_{E}\right)$ fails to have the Kadec-Klee property.

We remark finally that the principal results of this paper continue to hold in the more general setting of symmetric spaces of measurable operators affiliated with a semi-finite non-atomic von Neumann algebra. While some of these results have been announced in [CS2], the technical details are considerably more complicated and will appear elsewhere.

\section{REFERENCES}

[An] Anderson K.W., Midpoint Local Uniform Convexity, Dissertation, Univ. of Illinois, 1960.

[Ar] Arazy J., More on convergence in unitary matrix spaces, Proc. Amer. Math. Soc. 83 (1981), 44-48. MR 82f:46009 
[Bi] Birkhoff G., Lattice theory, A. M. S. Colloquium Publications, 3rd ed., vol. XXV, 1967. MR 37:2638

[BS] Bennett C. and Sharpley R., Interpolation of Operators, Academic Press, 1988. MR 89e: 46001

[CS1] Chilin V.I. and Sukochev F.A., Convergence in the measure topology in regular noncommutative symmetric spaces, Izv. VUZov. Mat. 9 (1990), 63-70, (Russian). MR 92g: 46081

[CS2] Chilin V.I. and Sukochev F.A., Symmetric spaces on semifinite von Neumann algebras, Soviet Math. Doklady 42 (1991), 97-101. MR 92a:46075

[DGL] Davis W.J., Ghoussoub N. and Lindenstrauss J., A lattice renorming theorem and applications to vector-valued processes, Trans. Amer. Math. Soc. 263 (1981), 531-540. MR 83d:46023

[DDDLS] P.G. Dodds, T.K. Dodds, C.J. Lennard, P. Dowling and F.A. Sukochev, A uniform Kadec-Klee property for symmetric operator spaces, Math. Proc. Camb. Phil. Soc. 118 (1995), 487-502. CMP 95:16

[Fr] Fremlin D.H., Stable subspaces of $L^{1}+L^{\infty}$, Math. Proc. Cambridge Phil. Soc. 64 (1968), 625-643. MR 37:749

[Go] Godefroy G., Seminaire Initiation a l'Analyse, Annee, vol. 23, 1982/83, pp. 1-6.

[Gr] Grothendieck A., Topological Vector Spaces, Gordon and Breach, 1973. MR 51:8772

[Ka1] Kadeč M.I., On weak and norm convergence, Dokl. Akad. Nauk SSSR 122 (1958), 13-16, (Russian). MR 20:5422

[Ka2] Kadeč M.I., On the connection between some rotundity properties of the unit ball of a Banach space, Funct. Anal. i Prilozhen. 16 (1982), 58-60, (Russian).

[KA] Kantorovich L.V. and Akilov G.P., Functional Analysis, Moscow: Nauka, 1972, (Russian).

[KPS] Krein S.G., Petunin Ju.I., Semenov E.M., Interpolation of Linear Operators, Moscow: Nauka, 1978, (Russian). MR 81f:46086

[Lu] Luxemburg W.A.J., Rearrangement invariant Banach function spaces, Queen's Papers in Pure and Applied Mathematics 10 (1967), 83-144.

[MS] Medzhitov A., Sukochev F.A., The property (H) in Orlicz spaces, Bull. Polish Acad. Sci. 40 (1992), 5-11.

[Ra] Radon J., Theorie und Anwendungen der absolut additiven Mengenfunctionen, Sitz. Akad. Wiss. Wien 122 (1913), 1295-1438.

[Ri1] Riesz F., Sur la convergence en moyenne I, Acta Sci.Math. 4 (1928/29), 58-64.

[Ri2] Riesz F., Sur la convergence en moyenne II, Acta Sci.Math. 4 (1928/29), 182-185.

[Se1] Sedaev A.A., On the (H)-property in symmetric spaces, Teoriya Funkcii, Funk. Anal. i Prilozenia 11 (1970), 67-80, (Russian). MR 45:4140

[Se2] Sedaev A.A., On weak and norm convergence in interpolation spaces, Trudy 6 zimney shkoly po mat. programm. i smezn. voprosam., Moscow, 1975, pp. 245-267, (Russian). MR 58:12426

[Sm] Smith M., Some examples concerning rotundity in Banach spaces, Math. Ann. 233 (1978), 155-161. MR 58:2168

[Su] Sukochev F.A., The property (MLUR) in symmetric (KB)-spaces, Mat. Zametki 52 (1992), 149-151, (Russian); [English translation in: Mathematical Notes, vol. 52, 1992, pp. 1280-1282]. MR 94c: 46030

(V. I. Chilin) Department of Mathematics, Tashkent State University, Tashkent 700095, UZBEKISTAN

(P. G. Dodds and F. A. Sukochev) Department of Mathematics and Statistics, The Flinders University of South Australia, GPO Box 2100, Adelaide, SA 5001, Australia

E-mail address: peter@ist.flinders.edu.au

E-mail address: sukochev@ist.flinders.edu.au

(A. A. Sedaev) Department of Mathematics, Voronež Civil Engineering Institute, VORONEŽ, 394000, RussiA 